\title{
Lattice QCD Simulations in External Background Fields
}

\author{
Massimo D'Elia
}

\begin{abstract}
We discuss recent results and future prospects regarding the investigation, by lattice simulations, of the non-perturbative properties of QCD and of its phase diagram in presence of magnetic or chromomagnetic background fields. After a brief introduction to the formulation of lattice QCD in presence of external fields, we focus on studies regarding the effects of external fields on chiral symmetry breaking, on its restoration at finite temperature and on deconfinement. We conclude with a few comments regarding the effects of electromagnetic background fields on gluodynamics.
\end{abstract}

\section{Introduction}

The properties of strong interactions in presence of strong external fields are of great phenomenological and theoretical importance. Large magnetic or chromomagnetic background fields, of the order of $10^{16}$ Tesla, i.e. $\sqrt{|e| B} \sim 1.5 \mathrm{GeV}$, may have been produced at the time of the cosmological electroweak phase transition [1] and may have influenced the subsequent evolution of the Universe, in particular the transition from deconfined to confined strongly interacting matter. Slightly lower magnetic fields are expected to be produced in non-central heavy ion collisions (up to $\sim 10^{15}$ Tesla at LHC [2, 3]), where they may give rise, in presence of non-trivial topological vacuum fluctuations, to $\mathrm{CP}$-odd effects consisting in the separation of electric charge along the direction of the background magnetic field, the so-called chiral magnetic effect [4, 2, 5]. Finally, large magnetic (or even chromomagnetic [6]) fields are expected in some compact astrophysical objects, such as magnetars [7] (see [8] for a recent review on the subject).

Massimo D'Elia

Dipartimento di Fisica dell'Università di Pisa and INFN - Sezione di Pisa,

Largo Pontecorvo 3, I-56127 Pisa, Italy

e-mail:delia@df.unipi.it 
Apart from the phenomenological issues above, there is great interest also at a purely theoretical level, since background fields can be useful probes to ge insight into the properties of strong interactions and the non-perturbative structure of the QCD vacuum. A typical example is chiral symmetry breaking, which is predicted to be enhanced by the presence of a magnetic background, a phenomenon which is known as magnetic catalysis $[9,10,11,12,13,14,15,16,17,18,19,20,21,22,23$, $24,25,26,27,28,29,30,31,32,33,34,35,36,37]$.

Such interest justifies the large efforts which have been dedicated in the recent past to this subject by a variety of different approaches, most of which are reviewed in the present volume. Lattice QCD represents, in general, the ideal tool for a first principle investigation of the non-perturbative properties of strong interactions. This is particularly true for the study of QCD in presence of a strong external magnetic or chromomagnetic field, since no kind of technical problem, such as a sign problem, appears, preventing standard Monte-Carlo simulations, as it happens instead at finite baryon chemical potential.

Similarly to the continuum theory, the magnetic field is introduced on the lattice in terms of additional $U(1)$ degrees of freedom (see Sec. 2.17, which are not directly coupled to the original $S U(3)$ link variables and affect quark propagation by a modification of the covariant derivative (i.e. the fermion matrix). The fermion determinant, contrary to the case of a baryon chemical potential $\mu_{B}$ or of an electric background field, is still real and positive, allowing for a probabilistic interpretation of the path integral measure. Lattice studies including the presence of electromagnetic fields have been done since long, originally with the purpose of studying the electromagnetic properties of hadrons [38, 39, 40, 41, 42]. The introduction of a chromomagnetic field requires a different approach, since in this case the background variables are strictly related to the quantum gluon degrees of freedom: a standard procedure is that defined in the framework of the lattice Schrödinger functional [43, 44, 45, 46, 47, 48, 49, 50, 51] (see Sec. 2.2.).

The recent interest about external field effects in the QCD vacuum and around the deconfining transition has stimulated lattice investigations by many groups $[52$, 53, 54, 55, 56, 57, 58, 59, 60, 61, 62, 63, 64, 65, 66]. Several studies have followed a quenched or partially quenched approach, considering only the effect of the magnetic background on physical observables, i.e. on quarks propagating in the background of the given magnetic field and of non-Abelian gauge configurations, the latter being sampled without taking into account the magnetic field.

However, other studies have shown that the electromagnetic background can have, via quark loop effects, a strong influence also on the distribution of nonAbelian fields, thus requiring an unquenched approach. A considerable contribution to magnetic catalysis appears to be related to gluon field modifications [59]; the magnetic field clearly affects also gluonic observables, like the plaquette [63] or those typically related to confinement/deconfinement, like the Polyakov loop [57, 62. 63]. That claims for a more systematic investigation about the effects of electromagnetic backgrounds on the gluonic sector of QCD and about the possible interplay between magnetic and chromomagnetic backgrounds, which could have many 
implications both at a theoretical and at a phenomenological level (e.g. for cosmology or heavy ion collisions).

The review is organized as follows. In Sec. 2 we discuss the formulation of QCD in external fields, considering both the case of an electromagnetic and of a chromomagnetic field. In Sec. 3 we review studies regarding vacuum properties in external fields, in particular concerning magnetic catalysis. Sec. 团is devoted to a discussion about the influence of magnetic or chromomagnetic background fields on deconfinement and chiral symmetry restoration. In Sec.5 5 we extend the discussion about the influence that electromagnetic fields may have on the gluonic sector, with a particular emphasys on symmetries and on suggestions for future studies on the subject.

\section{Background Fields on the Lattice}

In this Section we will briefly review the methods which are commonly adopted for a numerical study of QCD in presence of background fields. There is a difference between the case of electromagnetic and chromomagnetic backgrounds: in the former, the external field simply adds new $U(1)$ degrees of freedom which contribute to quark propagation but are decoupled from the $S U(3)$ degrees of freedom and from their updating during the Monte-Carlo simulation; in the latter, the background field is made up of the same degrees of freedom which are dynamically updated during the simulation, i.e. the $S U(3)$ gauge variables, and one has to follow a Schrödinger functional approach.

\subsection{Electromagnetic Fields}

A quark field $q$ propagating in the background of a non-abelian $S U(3)$ gauge field plus an electromagnetic field is described, in the Euclidean space-time, by a Lagrangean $\bar{\psi}(\not D+m) \psi$, where the covariant derivative

$$
D_{\mu}=\partial_{\mu}+\mathrm{i} g A_{\mu}^{a} T^{a}+\mathrm{i} q a_{\mu}
$$

contains contributions from the non-Abelian gauge field $A_{\mu}=A_{\mu}^{a} T^{a}$ and from the Abelian gauge field $a_{\mu}$. Here $T^{a}$ are the $S U(3)$ generators, $g$ is the $S U(3)$ gauge coupling and $q$ is the quark electric charge.

Going to a discrete lattice formulation, $S U(3)$ gauge invariance is naturally preserved [67] by requiring that quarks pick up an appropriate non-Abelian phase when hopping from one site of the lattice to the other, so that the gauge field $A_{\mu}$ is substituted by the elementary parallel transports $U_{\mu}(n)$, which reduce to $U_{\mu}(n) \simeq 1+\mathrm{i} g a A_{\mu}(n)$ as the lattice spacing $a$ vanishes in the continuum limit.

A gauge invariant quantity involving an antiquark at site $n$ and a quark at site $n+\hat{\mu}$ is therefore $\bar{\psi}(n) U_{\mu}(n) \psi(n+\hat{\mu})(\hat{\mu}$ is a unit vector on the lattice). The 
same approach can be taken for the Abelian field, so that quarks going from site $n$ to site $n+\mu$ pick up also the Abelian phase $u_{\mu}(n)=\exp \left(\mathrm{i} q \int_{a n}^{a(n+\hat{\mu})} d x_{\mu} a_{\mu}\right) \simeq$ $\exp \left(\mathrm{i} e q a_{\mu}(n)\right)$. A possible discretization of the covariant derivative is then

$$
D_{\mu} \psi \rightarrow \frac{1}{2 a}\left(U_{\mu}(n) u_{\mu}(n) \psi(n+\hat{\mu})-U_{\mu}^{\dagger}(n-\hat{\mu}) u_{\mu}^{*}(n-\hat{\mu}) \psi(n-\hat{\mu})\right)
$$

Of course, in presence of different quark species, carrying different electric charges $q$, the $U(1)$ part of the covariant derivative, $u_{\mu}(n)$, will change from quark to quark. Therefore, as usual, the discretized version of the fermion action is a bilinear form in the fermion fields, $\bar{\psi}(i) M_{i, j} \psi(j)$, however the elements of $M$ now belong to $U(3)$ instead of $S U(3)$.

\subsubsection{Magnetic Fields}

To take an explicit example, which is directly linked to most of the results that we discuss in the following, let us consider the finite temperature partition function of two degenerate quarks, e.g. the $u$ and $d$ quarks, in presence of a constant and uniform magnetic background field. We shall consider a rooted staggered fermion discretized version of the theory, in which each quark is described by the fourth root of the determinant of a staggered fermion matrix:

$$
Z(T, B) \equiv \int \mathscr{D} U e^{-S_{G}} \operatorname{det} M^{\frac{1}{4}}\left[B, q_{u}\right] \operatorname{det} M^{\frac{1}{4}}\left[B, q_{d}\right]
$$

where in the standard formulation

$$
\begin{aligned}
M_{i, j}[B, q]=a m \delta_{i, j} & +\frac{1}{2} \sum_{v=1}^{4} \eta_{v}(i)\left(u_{v}(B, q)(i) U_{v}(i) \delta_{i, j-\hat{v}}\right. \\
& \left.-u_{v}^{*}(B, q)(i-\hat{v}) U_{v}^{\dagger}(i-\hat{v}) \delta_{i, j+\hat{v}}\right) .
\end{aligned}
$$

$\mathscr{D} U$ is the functional integration over the non-Abelian gauge link variables, $S_{G}$ is the discretized pure gauge action and $u(B, q)_{i, v}$ are the Abelian links corresponding to the background field; $i$ and $j$ refer to lattice sites and $\eta_{i, v}$ are the staggered phases. We shall consider two different charges for the two flavors, $q_{u}=2|e| / 3$ and $q_{d}=-|e| / 3$. Periodic or antiperiodic boundary conditions along the Euclidean time direction must be taken, respectively for gauge or fermion fields, in order to define a thermal theory: the temperature $T$ corresponds to the inverse temporal extension.

A special discussion must be devoted to the issue of spatial boundary conditions. It is well known that, for a given lattice size (which is usually constrained by the computational power available) periodic boundary conditions in space (for all fields) are the one which best approximate the infinite volume limit, and are therefore the standard choice in lattice simulations. On the other hand, they place some constraints on the possible magnetic fields [68, 69, 70, 71]. 
Let us consider a magnetic field directed along the $\hat{z}$ axis of a three-dimensional torus, $\mathbf{B}=B \hat{z}$, and let $l_{x}$ and $l_{y}$ be the torus extensions in the orthogonal directions. The circulation of $a_{\mu}$ along any closed path in the $x-y$ plane, enclosing a region of area $A$, is given by Stokes theorem

$$
\oint a_{\mu} d x_{\mu}=A B
$$

On the other hand it is ambiguous, on a closed surface like a torus, to state which is the region enclosed by a given path: we can choose the complementary region of area $l_{x} l_{y}-A$ and equally state

$$
\oint a_{\mu} d x_{\mu}=\left(A-l_{x} l_{y}\right) B
$$

The ambiguity can be resolved either by admitting discontinuities for $a_{\mu}$ somewhere on the torus, or by covering the torus with various patches corresponding to different gauge choices. However it is essential to guarantee that the ambiguity be not visible by any charged particle moving on the torus, i.e. the phase factor picked up by a particle of charge $q$ moving along the given path must be a well defined quantity. Such requirement leads to magnetic field quantization, indeed it is satisfied only if

$$
\exp (\mathrm{i} q B A)=\exp \left(\mathrm{i} q B\left(A-l_{x} l_{y}\right)\right)
$$

i.e. if

$$
q B=\frac{2 \pi b}{l_{x} l_{y}}
$$

where $b$ is an integer. It is interesting to notice that this is exactly the same argument leading to Dirac quantization of the magnetic monopole charge (in that case the closed surface is any sphere centered around the monopole). The quantization rule depends on the electric charges of the particles moving on the torus 1 , in our case it is set by the $d$ quark, which brings the smallest charge unit $q_{d}=-|e| / 3$, hence

$$
|e| B=\frac{6 \pi b}{l_{x} l_{y}}=\frac{6 \pi b}{a^{2} L_{x} L_{y}}
$$

where $L_{x}$ and $L_{y}$ are the dimensionless lattice extensions in the $x, y$ directions.

A possible choice for the gauge links, corresponding to the continuum gauge field

$$
a_{y}=B x ; \quad a_{\mu}=0 \text { for } \quad \mu=x, z, t .
$$

is the following:

1 Of course we assume all particles living on the torus to carry integer multiples of some elementary electric charge, otherwise a consistent quantization of the magnetic field would not be possible. 


$$
u_{y}(B, q)(n)=e^{\mathrm{i} a^{2} q B n_{x}} ; \quad u_{\mu}(B, q)(n)=1 \text { for } \mu=x, z, t
$$

The smoothness of the background field across the boundaries and the gauge invariance of the fermion action are guaranteed if appropriate boundary conditions are taken for the fermion fields along the $x$ direction [71], that corresponds to modifying the $U(1)$ gauge links in the $x$ direction as follows:

$$
\left.u_{x}(B, q)(n)\right|_{n_{x}=L_{x}}=e^{-\mathrm{i} a^{2} q L_{x} B n_{y}} .
$$

A different condition on the possible magnetic field values explorable on the lattice is placed by the ultraviolet (UV) cutoff, i.e. by discretization itself. All the information about the presence of the magnetic field is contained in the phase factors picked up by particles moving on the lattice. There is a minimum such path on a cubic lattice, the plaquette; in particular, a particle moving around a $x-y$ plaquette takes a phase factor

$$
\exp \left(\mathrm{i} q a^{2} B\right)=\exp \left(\mathrm{i} \frac{2 \pi b}{L_{x} L_{y}}\right)
$$

and all other possible phase factors, corresponding to different paths, are positive integer powers of that. It is evident that the phase factor in Eq. (13) cannot distinguish magnetic fields such that $q a^{2} B$ differs by multiples of $2 \pi$ : one would need smaller paths to do that, which are unavailable because of the UV cutoff. Hence it is possible to define a sort of first Brillouin zone for the magnetic field

$$
-\frac{\pi}{a^{2}}<q B<\frac{\pi}{a^{2}}
$$

and we expect that physical quantities must be periodic in $q B$ with a period $2 \pi / a^{2}$; such periodicity is unphysical and one should always be cautious when exploring magnetic field values which get close to the limiting values in Eq. (14).

Another issue which is relevant to the lattice implementation of background fields regards translational invariance. The magnetic field $B$ breaks the translational invariance of the continuum torus in the $x-y$ directions explicitly [71]. That is clearly seen by looking at the $U(1)$ Wilson lines (holonomies) $W_{x}$ and $W_{y}$, i.e. the $U(1)$ parallel transports along straight paths in the $x$ or $y$ direction and closed around the torus by periodic boundary conditions, which are equal to

$$
W_{x}=\exp \left(-\mathrm{i} q B l_{x} y\right) ; \quad W_{y}=\exp \left(\mathrm{i} q B l_{y} x\right)
$$

as can be verified explicitly by means of the elementary parallel transports given in Eq. (11) and (12). The Wilson lines therefore leave only a residual simmetry corresponding to discrete translations by multiples of

$$
\tilde{a}_{x}=\frac{2 \pi}{q B l_{y}}=\frac{l_{x}}{b} ; \quad \tilde{a}_{y}=\frac{2 \pi}{q B l_{x}}=\frac{l_{y}}{b} ;
$$


note that Wilson lines are gauge invariant quantities, hence the breaking of translational invariance is not a matter of gauge choice.

However, on a discrete cubic lattice, translational invariance is already broken to a discrete residual group, corresponding to multiples of the lattice spacing $a$, hence for a lattice theory in presence of a constant magnetic field, translational invariance is further reduced to discrete steps which are multiples of both $\tilde{a}_{x / y}$ and $a$. Since $a / \tilde{a}_{x / y}=b / L_{x / y}$, lattice translational symmetry is preserved for $b$ multiple of $L_{x / y}$, but is strongly reduced or completely lost for different values, despite the fact that the magnetic background field is uniform.

We have discussed so far about one possible lattice implementation of the fermionic action in presence of a magnetic field, Eqs. (2) and (4), which is based on the simplest symmetric discretization of the covariant derivative, which takes quarks from nearest neighbours sites, rotating them by a non-Abelian and an Abelian phase, both corresponding to the simplest straight path, i.e. the elementary parallel transport. Such kind of discretization has been adopted in many lattice studies, see e.g. [57, 59, 63].

It is well known, however, that different, improved discretizations can lead to an improved convergence towards the continuum limit. Improvement can proceed in different ways: one can consider less simple discretizations of the derivative, involving fermion fields which are not nearest neighbours; one can also consider and average over paths different from the simplest straight one, so as to smear out UV fluctuations: this is idea adopted, for instance, in [62, 65, 66], where stout smearing has been used. If one considers lattice derivatives involving non-nearest neighbours lattice sites, one must include appropriate composite $U(1)$ parallel transports, so as to preserve the $U(1)$ gauge invariance of the fermion action. Which paths, however, and how the chosen paths are related to the paths considered for the non-Abelian phases, is a question which leaves much freedom.

Let us consider, for simplicity, a gauge invariant bilinear term involving nearest neighbour quarks, $\bar{\psi}(n) U_{\mu}(n) \psi(n+\hat{\mu})$, which reduces to $\bar{\psi}(x)\left(1+a D_{\mu}\right) \psi(x)+$ $O\left(a^{2}\right)$ in the naïve continuum limit. We can choose a more general, improved such term, involving different paths, i.e. we can modify

$$
\bar{\psi}(n) U_{\mu}(n) \psi(n+\hat{\mu}) \rightarrow \bar{\psi}(n)\left(\sum_{C(n, n+\hat{\mu})} \alpha_{C} U_{C}\right) \psi(n+\hat{\mu})
$$

where $C(n, n+\hat{\mu})$ are a set of paths connecting $n$ to $n+\hat{\mu}$ (e.g. for smearing one includes staples), $U_{C}$ are the parallel transports taken along those paths and the coefficients $\alpha_{C}$ are chosen in order to keep the naïve continuum limit unchanged, up to $O\left(a^{2}\right)$. Let us now consider the inclusion of a $U(1)$ external field: a possible prescription is that, as quarks explore all considered paths $C$, taking the associated non-Abelian phases, they also take the corresponding $U(1)$ phases along the same paths, i.e. 


$$
\bar{\psi}(n) U_{\mu}(n) u_{\mu}(n) \psi(n+\hat{\mu}) \rightarrow \bar{\psi}(n)\left(\sum_{C(n, n+\hat{\mu})} \alpha_{C} U_{C} u_{C}\right) \psi(n+\hat{\mu}) .
$$

However, different prescriptions can be taken and, in general, the sum over $U(1)$ phases can be decoupled from that on $S U(3)$ phases, without affecting gauge invariance. We can consider for example

$$
\bar{\psi}(n)\left(\sum_{C(n, n+\hat{\mu})} \alpha_{C} U_{C}\right)\left(\sum_{C^{\prime}(n, n+\hat{\mu})} \alpha_{C^{\prime}}^{\prime} u_{C}^{\prime}\right) \psi(n+\hat{\mu})
$$

where $C^{\prime}$ is a different set of paths. This is the choice made, for instance, in 62 , 65, 66], where stout smearing is applied to $S U(3)$ links, while the $U(1)$ phases are left unchanged (i.e. $C^{\prime}$ runs over the elementary straight path only); that seems a reasonable choice, since the $U(1)$ field is already a classical smooth field.

It can be easily shown that for a uniform background and for free fermions, i.e. in absence of non-Abelian fields, all possible different choices of paths for the $U(1)$ term are equivalent, apart from a global phase stemming from interference (Aharonov-Bohm like) effects among the different paths. In presence of nonAbelian fields, however, this is in general not true and it would be interesting to explore the systematics connected to different choices.

A final comment concerns the issue of possible renormalizations related to the introduction of the external magnetic field. The quantity entering the covariant derivative and quark dynamics is the combination $q a_{\mu}$, which does not renormalize. The situation is clearer in the lattice formulation, where the objects carrying information about the external field are the parallel transports of the $U(1)$ gauge field along closed loops (think of the loop expansion of the fermionic determinant). Such phase factors are gauge inviariant quantities and cannot renormalize. The only remaining

possibility is that the lattice spacing $a$ itself depends on the magnetic field $B$, because of external field effects at the scale of the UV cutoff, so that the physical area enclosed by a given loop changes, leading to an effective external field renormalization. Such possibility, however, has been excluded by a detailed study presented in [62], showing that $a$ does not depend on $B$ within errors.

\subsubsection{Electric Fields}

After the inclusion of a background magnetic field, the $\not D$ operator is still antiHermitean and it still anticommutes with $\gamma_{5}$, hence its spectrum is purely imaginary with non-zero eigenvalues coming in conjugate pairs, so that $\operatorname{det}(\not D+m)>0$ and Monte-Carlo simulations are feasible, i.e. the path integral measure can be interpreted as a probability distribution function over gauge configurations.

The situation is different in presence of background electric fields [72, 42, 41]. Let us consider a constant electric field $\mathbf{E}=E \hat{z}$, there are various possible choices 
for a corresponding vector potential in Minkowski space, like $\left(A_{t}=-E z, A_{i}=0\right)$ or $\left(A_{z}=-E t, A_{x}=A_{y}=A_{t}=0\right)$. In every case, after continuation to Euclidean space time, $t \rightarrow-\mathrm{i} \tau$ and $A_{t} \rightarrow \mathrm{i} A_{0}$, the vector potential becomes purely imaginary, thus destroying the anti-Hermitean properties of the Dirac matrix.

That means that the fermion determinant is complex and numerical simulations are not feasible. This "sign problem" has a very strict relation with the usual sign problem which is encountered in the study of QCD at finite baryon chemical potential, indeed also a baryon chemical potential can be viewed as a constant background potential $A_{0} \neq 0$. As for finite density QCD, also in this case a possible way out is to consider a purely imaginary electric field: the lattice formulation is then completely analogous to that of a magnetic field. For example, a purely imaginary electric field along the $\hat{z}$ direction can be formulated on the lattice following Eqs. (11) and (12), by just replacing replacing $(x, y) \rightarrow(z, t)$ and $B \rightarrow \operatorname{Im}(E)$. A similar approach, followed by analytic continuation to real electric fields, is that usually adopted for the study of hadron electric polarizabilities.

\subsection{Chromomagnetic Background Fields}

As we have discussed above, the implementation of an electromagnetic background field amounts to adding extra $U(1)$ degrees of freedom to the Dirac matrix: the added $U(1)$ field is a classic, static field which is decoupled from the $S U(3)$ gauge field appearing in the functional integration. The situation is different for a color background field, since in this case one would like to consider quantum fluctuations of the non-Abelian field around a given backgroud, e.g. a static ad uniform chromomagnetic field, however the functional integration over gauge variables can destroy information about the background field completely.

A common way to approach the problem is based on the Schrödinger functional approach [43, 44]: one considers functional integration over Euclidean space-time with fixed temporal boundaries, $\tau_{1}$ and $\tau_{2}$, and over gauge configurations which are frozen to particular assigned values, $A_{i}^{\text {ext } 1}\left(\mathbf{x}, \tau_{1}\right)$ and $A_{i}^{\text {ext } 2}\left(\mathbf{x}, \tau_{2}\right)$, at the initial and final times. This is related to the quantum amplitude of passing from the field eigenstate $\left|A_{i}^{\text {ext } 1}\right\rangle$ to the field eigenstate $\left|A_{i}^{\text {ext } 2}\right\rangle$ in the time $\left(\tau_{2}-\tau_{1}\right)$. The amplitude is dominated, in the classical limit, by the gauge configuration which has the minimal action among those with the given boundary conditions; functional integration can then be viewed as an integration over quantum fluctuations around this classical background field.

In Refs. [45, 46, 47, 48, 49] such formalism has been implemented on the lattice by considering equal initial and final fields, $A_{i}^{\text {ext } 1}\left(\mathbf{x}, \tau_{1}\right)=A_{i}^{\text {ext2 }}\left(\mathbf{x}, \tau_{2}\right)=A_{i}^{\text {ext }}(\mathbf{x})$. In

this way one can define a lattice gauge invariant effective action $\Gamma\left[\mathbf{A}^{\mathrm{ext}}\right]$ for the external background field $\mathbf{A}^{\text {ext. }}$.

$$
\Gamma\left[\mathbf{A}^{\mathrm{ext}}\right]=-\frac{1}{L_{t}} \ln \left\{\frac{\mathscr{Z}\left[\mathbf{A}^{\mathrm{ext}}\right]}{\mathscr{Z}[0]}\right\}
$$


where $L_{t}$ is the lattice temporal extension and $\mathscr{Z}\left[\mathbf{A}^{\mathrm{ext}}\right]$ is the lattice functional integral

$$
\begin{aligned}
\mathscr{Z}_{T}\left[\mathbf{A}^{\mathrm{ext}}\right] & =\int_{U_{k}\left(L_{t}, \mathbf{x}\right)=U_{k}(0, \mathbf{x})=U_{k}^{\mathrm{ext}}(\mathbf{x})} \mathscr{D} U \mathscr{D} \psi \mathscr{D} \bar{\psi} e^{-\left(S_{W}+S_{F}\right)} \\
& =\int_{U_{k}\left(L_{t}, \mathbf{x}\right)=U_{k}(0, \mathbf{x})=U_{k}^{\operatorname{ext}}(\mathbf{x})} \mathscr{D} U e^{-S_{W}} \operatorname{det} M
\end{aligned}
$$

where $S_{G}$ and $S_{F}$ are the pure gauge and fermion action respectively, $M$ is the fermionic matrix. The functional integration is performed over the lattice links, with the constraint

$$
U_{k}\left(\mathbf{x}, x_{t}=0\right)=U_{k}\left(\mathbf{x}, x_{t}=L_{t}\right)=U_{k}^{\mathrm{ext}}(\mathbf{x}), \quad(k=1,2,3),
$$

where $U_{k}^{\text {ext }}(\mathbf{x})$ are the elementary parallel transports corresponding to the continuum gauge potential $\mathbf{A}^{\mathrm{ext}}(x)=\mathbf{A}_{a}^{\mathrm{ext}}(x) \lambda_{a} / 2$; fermion fields as well as temporal links are left unconstrained. $\mathscr{Z}[0]$ is defined as in Eq. (21), but adopting a zero external field.

In the following we shall consider the case of an Abelian static and uniform chromomagnetic background field: in this case one can choose $\lambda_{a}$ belonging to the Cartan subalgebra of the gauge group, e.g. $\lambda_{a}=\lambda_{3}$, while the explicit form of $\mathbf{A}_{a}^{\mathrm{ext}}(x)$, hence of the lattice links, can be chosen in the same way as for a standard magnetic field, see Eqs. (10), (11) and (12). In this case, as well as in other cases in which the static background field does not vanish at spatial infinity, one usually imposes also that spatial links exiting from sites belonging to the spatial boundaries are fixed, at all times, according to Eq. (22): that corresponds to the requirement that fluctuations over the background field vanish at infinity.

Notice that a colored background field influences directly, and through the same gauge coupling $g$, the dynamics of both quark and gluon fields. Indeed numerical simulations in presence of colored background fields have considered originally just the case of pure Yang-Mills theories.

Differently from the usual formulation of the lattice Schrödinger functional 43, 44], where a cylindrical geometry is adopted, the effective action defined by Eq. (20) assumes an hypertoroidal geometry, i.e. the first and the last time slice are identified and periodic boundary conditions are assumed in the time direction for gluon fields. For finite values of $L_{t}$, having adopted also the prescription of anti-periodic boundary conditions in time direction for quark fields, Eq. (21) can be interpreted as the thermal partition function $\mathscr{Z}_{T}\left[\mathbf{A}^{\text {ext }}\right]$ in presence of the background field $\mathbf{A}^{\text {ext }}$, with the temperature given by $T=1 /\left(a L_{t}\right)$. The gauge invariant effective action, Eq. (20), is then replaced by the free energy functional:

$$
\mathscr{F}\left[\mathbf{A}^{\mathrm{ext}}\right]=-\frac{1}{L_{t}} \ln \left\{\frac{\mathscr{Z}_{T}\left[\mathbf{A}^{\mathrm{ext}}\right]}{\mathscr{Z}_{T}[0]}\right\} .
$$




\section{Vacuum Properties in Backgroud Fieds: Magnetic Catalysis}

One of the most significant effects that a magnetic background field can induce on the QCD vacuum, as well as on other systems characterized by the chiral properties of fermion fields, is known as magnetic catalysis. It consists in an enhancement of chiral symmetry breaking, or spontaneous mass generation, which can be thought as related to the dimensional reduction taking place in the dynamics of particles moving in a strong external magnetic field (see [35] for a recent comprehensive review).

The enhancement of chiral symmetry breaking reveals itself in a dependence of the chiral condensate $\langle\bar{\psi} \psi\rangle$ on the magnetic field $B$; there are several predictions for the actual functional form $\langle\bar{\psi} \psi\rangle(B)$, depending on the adopted model, however some general features can be summarized as follows. By charge conjugation symmetry, $\langle\bar{\psi} \psi\rangle$ must be an even function of $B$, hence, if the theory is analytic around $B=0$, one expects that $\langle\bar{\psi} \psi\rangle$ depends quadratically on $B$ in a regime of small enough fields. The analyticity assumption is violated in presence of charged massless fermions, indeed chiral perturbation theory [19] predicts a linear behavior in $B$ if $m_{\pi}=0$; such linear dependence is recovered in computations at $m_{\pi} \neq 0$, in the limit $e B \gg m_{\pi}^{2}$ [20]. Different power behaviors in $B$ can be found in other model computations. In the following we shall make use of the relative increment of the chiral condensate, defined as

$$
r(B)=\frac{\langle\bar{\psi} \psi\rangle(B)-\langle\bar{\psi} \psi\rangle(B=0)}{\langle\bar{\psi} \psi\rangle(B=0)} .
$$

Early lattice investigations of magnetic catalysis have been done in the quenched approximation, both for $S U(2)$ [53] and for $S U(3)$ [58] gauge theories. In this case, the chiral condensate is computed by inverting a Dirac operator which contains the contribution from the external field, but on gauge configurations sampled in absence of dynamical fermion contributions. The outcome is that $r(B) \propto B$ for $S U(2)$ [53], while $r(B) \propto B^{v}$, with $v \sim 1.6$, for $S U(3)$ [58].

On the other hand, the inclusion of contributions from dynamical fermions moving in the background field may produce significant effects. The chiral condensate is related, via the Banks - Casher relation [73], to the density $\rho(\lambda)$ of eigenvalues of the Dirac operator around $\lambda=0:\langle\bar{\psi} \psi\rangle=\pi \rho(0)$. Such density can change, as a function of $B$, both because the Dirac operator definition itself changes, and because the distribution of gauge configurations, over which the operator is computed, is modified by dynamical fermion contributions. Studies of magnetic catalysis involving full dynamical simulations have been reported in [59, 65], for QCD with 3 colors, and in [63] for QCD with 2 colors.

In Ref. [59], where a standard staggered fermion and plaquette gauge discretization has been adopted, corresponding to a pseudo-Goldstone pion mass $m_{\pi} \sim 200 \mathrm{MeV}$, an attempt has been made to separate the contributions to magnetic catalysis coming from the modification of the Dirac operator from those coming from the modified gauge field distribution: such contributions have been named 


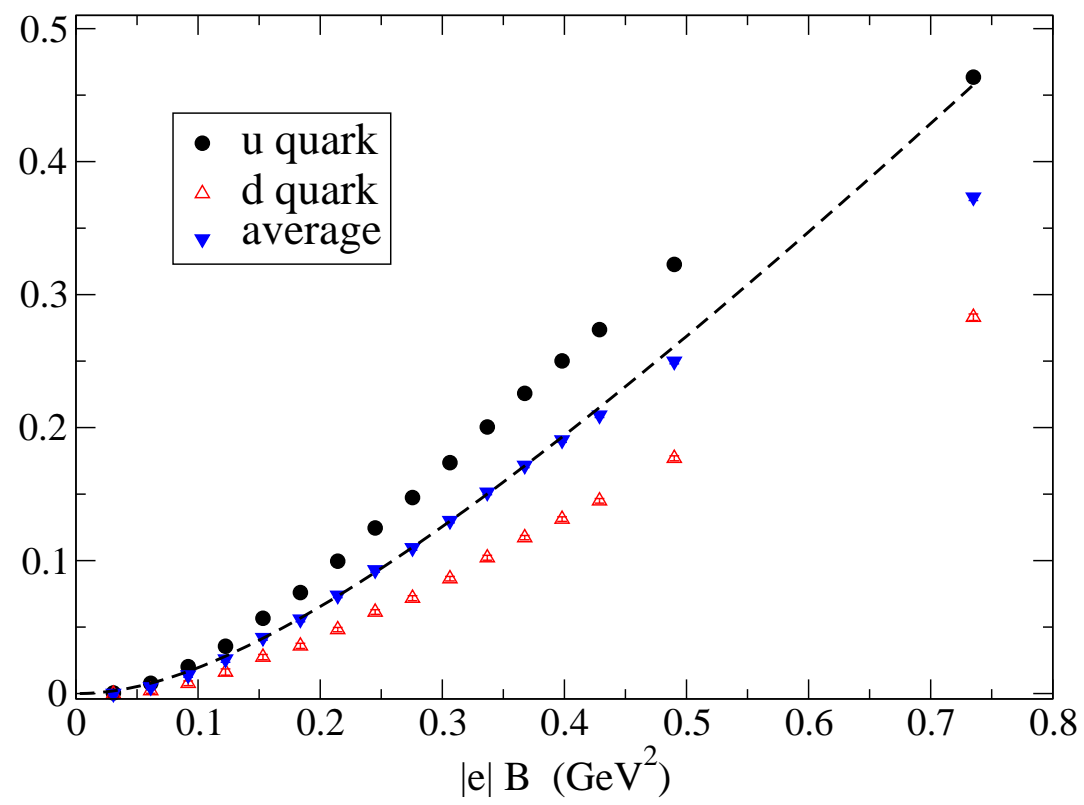

Fig. 1 We show the relative increment $r(B)$ (see Eq. 24p) as a function of $|e| B$ for $N_{f}=2$ QCD with $m_{\pi} \sim 200 \mathrm{MeV}$. Data are reported separately for the $u$ and $d$ quark condensates, as well as for their average, together with a best fit according to the functional dependence inspired by chiral perturbation theory [20]. Figure taken from [59].

"valence" and "dynamical", respectively. One can show that such separation can be done consistently in the limit of small external fields. Indeed, let us define the quantities

$$
\begin{gathered}
\langle\bar{\psi} \psi\rangle_{u / d}^{v a l}(B) \equiv \int \mathscr{D} U \mathscr{P}[m, U, 0] \operatorname{Tr}\left(M^{-1}\left[m, B, q_{u / d}\right]\right) \\
\langle\bar{\psi} \psi\rangle_{u / d}^{d y n}(B) \equiv \int \mathscr{D} U \mathscr{P}[m, U, B] \operatorname{Tr}\left(M^{-1}\left[m, 0, q_{u / d}\right]\right)
\end{gathered}
$$

and, from those, the average (over flavor) quantities $\langle\bar{\psi} \psi\rangle^{v a l}$ and $\langle\bar{\psi} \psi\rangle^{d y n} ; \mathscr{P}$ is the probability distribution for gauge fields (including quark loop effects) and $M$ is the fermion matrix. In the first case, one looks at the spectrum of the fermion matrix which includes the magnetic field explicitly, but is defined on non-Abelian configurations sampled with a measure $\mathscr{D} U \mathscr{P}[m, U, 0]$ which, even including dynamical fermion contributions, is taken at $B=0$ (partial quenching). In the second case the measure term takes into account the external field, which is however neglected in the 
definition of the fermion matrix. From $\langle\bar{\psi} \psi\rangle^{v a l}(B)$ and $\langle\bar{\psi} \psi\rangle^{d y n}(B)$ we can define the corresponding quantities $r^{v a l / d y n}$.

In the limit of small fields, $B$ acts as a perturbation for both the measure term $\mathscr{P}[m, U, B]$ and the observable $\operatorname{Tr}\left(M^{-1}\left[m, B, q_{u / d}\right]\right)$ : assuming quadratic corrections in $B$ (however this is not an essential assumption), one can write, configuration by configuration:

$$
\mathscr{P}[m, U, B]=\mathscr{P}[m, U, 0]+C B^{2}+O\left(B^{4}\right)
$$

and

$$
\operatorname{Tr}\left(M^{-1}[B]\right)=\operatorname{Tr}\left(M^{-1}[0]\right)+C^{\prime} B^{2}+O\left(B^{4}\right)
$$

where $C$ and $C^{\prime}$ depend in general on the quark mass and on the chosen configuration. Putting together the two expansions, one obtains [59]

$$
r(B)=r^{v a l}(B)+r^{d y n}(B)+O\left(B^{4}\right),
$$

therefore, at least in the limit of small fields, the separation of magnetic catalysis in a valence and a dynamical contribution is a well defined concept.

In Fig. 10 we report data obtained in [59] for the relative increment of the $u$ and $d$ quark condensates and for their average, as a function of $|e| B$. Magnetic catalysis is quite larger for the $u$ quark with respect to the $d$ quark: this is expected on the basis of the larger electric charge of the $u$ quark. Regarding their average, one observes that a functional dependence inspired by chiral perturbation theory [20] fits well data, when taking into account the unphysical quark mass spectrum considered in [59]. A simple quadratic dependence describes well data in the regime of small background fields, approaching a linear behavior for larger fields, while saturation effects starts to be visible as $\sqrt{|e| B}$ reaches the scale of the UV cutoff, which is quite low for the lattice setup used in [59], $a^{-1} \sim 0.7 \mathrm{GeV}$. Regarding the separation into valence and dynamical contributions, Fig. 2] taken again from [59], shows that it is indeed well defined in a significant range of magnetic fields, where the two contributions are roughly additive, in the sense that their sum gives back the full signal, as expected at least in the limit of small fields. Moreover, from Fig. 2] one learns that the dynamical contribution is roughly $40 \%$ of the total signal, at least for the discretization and quark mass spectrum adopted in [59]: this is an important contribution, which is larger than other usual unquenching effects, which are typically of the order of $20 \%$, and reflects a significant modification in the distribution of gauge fields, induced by the magnetic background field, which should be further investigated by future studies.

A new investigation of magnetic catalysis for the QCD vacuum has been done recently [65], making use of an improved gauge and (stout) rooted staggered discretization of the theory: results for the average increment of the $u$ and $d$ quark condensates have been reported for $N_{f}=2+1$ flavors (i.e. including also strange quark contributions), adopting physical quark masses and after extrapolation to the continuum limit. Also in this case one observes, for the relative increment $r(B)$ (see 


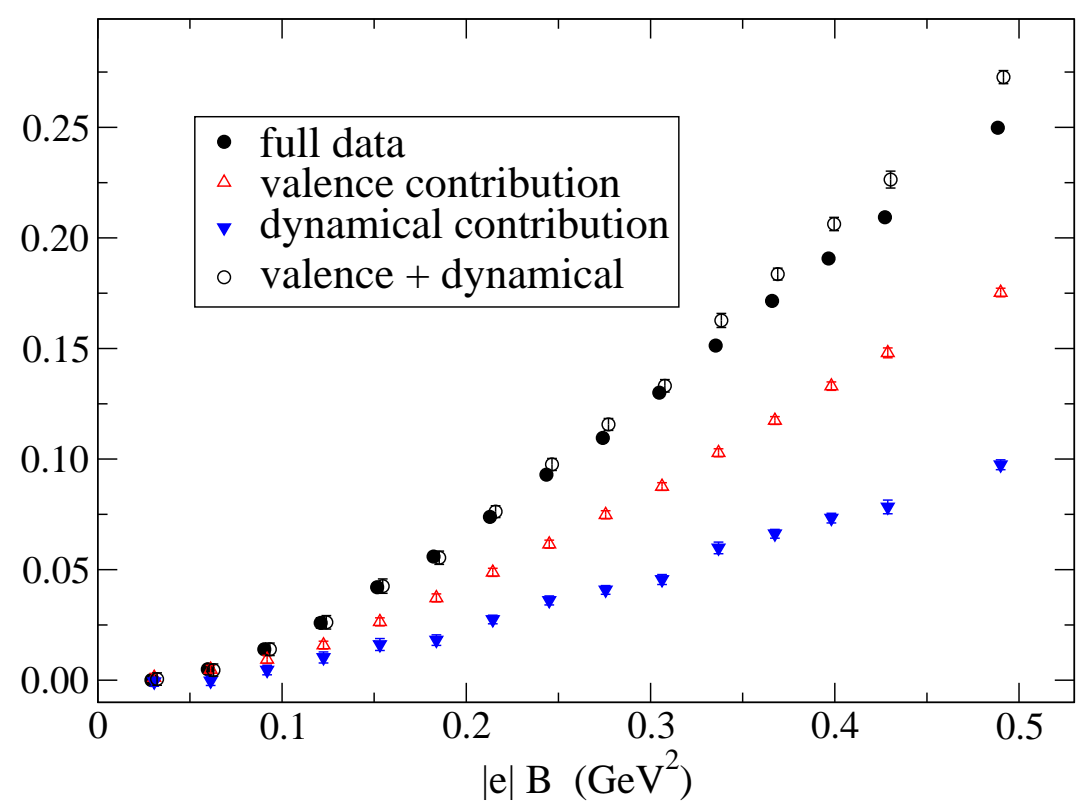

Fig. 2 Relative increment of the average of the $u$ and $d$ quark condensates as a function of the magnetic field. $r(B), r^{v a l}(B), r^{d y n}(B)$ and $r^{v a l}(B)+r^{d y n}(B)$ are reported separately. From [59].

Fig. 1 of Ref.[65]), a quadratic dependence on $|e| B$ for small external fields, followed by an almost linear dependence as $|e| B \gg m_{\pi}^{2}$; a nice agreement is found with predictions from chiral perturbation theory and from PNJL models [74], at least for not too large fields. It is interesting to notice that, in the regime of small fields, the results of [59] and those of [65] are compatible with each other if they are rescaled by a factor $m_{\pi}^{2}$ (which is different in the two studies2: the prediction from chiral perturbation theory is indeed, in the limit of small fields [20, 59]:

$$
r(B) \simeq \frac{(|e| B)^{2}}{96 \pi^{2} F_{\pi}^{2} m_{\pi}^{2}}
$$

The authors of [63], instead, have presented an investigation of magnetic catalysis for QCD with two colors and 4 staggered flavors, which are degenerate both in mass and electric charge: that permits, contrary to Refs. [59, 65], to avoid rooting and the possible systematic effects related to it. The lattice discretization is standard, as in [59], with also a similar range of pseudo-Goldstone pion masses. Results are qualitatively similar to those obtained in [59, 65], however the authors try also

\footnotetext{
2 The author thanks G. Endrodi for giving him access to the continuum extrapolated data of [65].
} 
an extrapolation to the chiral limit, thus verifying the prediction [19] for a linear dependence on $|e| B$ in such limit.

Till now we have discussed about magnetic catalysis in the QCD vacuum, i.e. in the low temperature, deconfined phase, where a consistent picture emerges, both from model and lattice computations. The situation is less clear as one approaches the high temperature, deconfined regime: results from [62, 65] have shown that the growth of the chiral condensate with the magnetic field may stop and even turn into an inverse magnetic catalysis at high enough temperatures; such effect may have a possible interpretation based on the effects of a strong magnetic field on gluodynamics (see [36, 35, 75] and the discussion in Section 5). Inverse magnetic catalysis has been predicted by some model computation [37], however in a regime of low temperature and high baryon density, which is different from that explored in [62, 65]; recently a possible explanation has been proposed according to which inverse catalysis derives from dimensional reduction induced by the magnetic field on neutral pions [76]. On the other hand, the lattice studies reported in [57, 63] do not show such effect, reporting instead for standard magnetic catalysis at all explored temperatures. We will comment on the possible origin of such discrepancies in the following Section, where we discuss about the effects of strong external fields on the QCD phase diagram.

Finally, among the studies aimed at clarifying the effects of strong magnetic fields on chiral dynamics, we mention those addressing the determination of the magnetic susceptibility of the chiral condensate, both in quenched [54, 58] and in unquenched QCD [66], which is part of the total contribution to the magnetic susceptibility of the QCD vacuum. In particular, recent unquenched results [66] show that such contribution is of diamagnetic nature.

\section{QCD Phase diagram in External Fields}

Similarly to what happens with other external parameters, like a finite baryon chemical potential, the introduction of a background field, either magnetic of chromomagnetic, can modify the phase structure of QCD. The interest in such issue is theoretical, on one side, since a background field can be viewed as yet another parameter of the QCD phase diagram, which can help in getting a deeper understanding of the dynamics underlying deconfinement and chiral symmetry restoration. There is however also great phenomenological interest, since strong background fields may be relevant to the cosmological QCD transition, to heavy ion collisions and to the physics of some compact astrophysical objects. The main questions regarding the QCD transition that one would like to answer can be summarized as follows:

1) do deconfinement and chiral symmetry restoration remain entangled, or is a strong enough magnetic field capable of splitting the two transitions?

2) does the (pseudo)critical temperature depend on the background field strength, and how?

3 ) does the nature of the transition depend on the background field strength? 


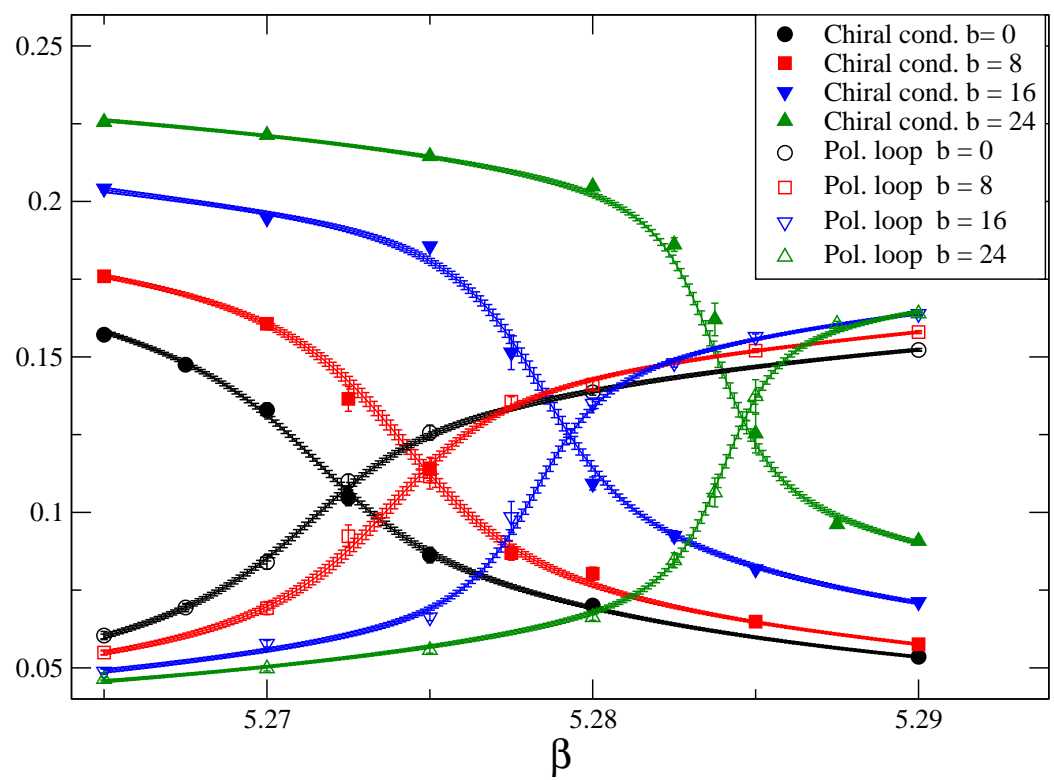

Fig. 3 Behavior of the chiral condensate and of the Polyakov loop, as a function of the inverse gauge coupling $\beta$ and for different magnetic field quanta $b$, on a $16^{3} \times 4$ lattice and for a pion mass $m_{\pi} \sim 200 \mathrm{MeV}$. Figure taken from [57].

4) does any new, unexpected phase of strongly interacting matter emerge for strong enough background fields?

Many model computations exist, which try to answer those questions [77, 78, 79, 80, 81, 82, 83, 84, 85, 86, 87, 88, 89, 90, 91, 92, 93, 94, 95, 96, in the following we will discuss results based on lattice simulations. The focus will be on aspects regarding deconfinement and chiral symmetry restoration, leaving aside other issues, like the possible emergence of a superconductive phase for strong enough magnetic fields [97, 60, 98, 99].

\subsection{Deconfinement Transition in a Strong Magnetic Background}

Investigating the effects of a background magnetic field on thermodynamics and on the phase diagram of QCD necessarily requires an approach in which the presence of the magnetic field is taken into account at the level of dynamical fermions.

A first study along these lines has been presented in [57], where finite temperature QCD with two degenerate flavors has been simulated in presence of a con- 
stant, uniform magnetic background. A standard rooted staggered discretization (see Eq. (3) ) and a plaquette gauge action have been adopted, with a lattice spacing of the order of $0.3 \mathrm{fm}$, and different quark masses, corresponding to a pseudo-Goldstone pion mass ranging from $200 \mathrm{MeV}$ to $480 \mathrm{MeV}$, with magnetic fields going up to $|e| B \sim 0.75 \mathrm{GeV}^{2}$.

Some results from [57] are reported in Figs. 3, 4, 5] and 6, In particular, Fig. 3 shows the behavior of the chiral condensate and of the Polyakov loop as a function of the inverse gauge coupling $\beta$, for the lowest pion mass explored $\left(m_{\pi} \sim 200\right.$ $\mathrm{MeV}$ ) and for various magnetic fields (expressed in units $b$ of the minimum quantum allowed by the periodic boundary conditions). Remember that the physical temperature, $T=1 / L_{t} a(\beta)$, is a monotonic, increasing function of $\beta$, and that, on the $16^{3} \times 4$ lattice explored in [57], the magnetic field is given by $|e| B=\left(3 \pi T^{2} / 8\right) b \sim$ $\left(0.03 \mathrm{GeV}^{2}\right) b$ around the transition.

Three facts are evident from Fig. 3. The chiral condensate increases, as a function of $B$, for all explored temperatures. The inflection points of the chiral condensate and of the Polyakov loop, signalling the location of the pseudo-critical temperature, move together towards higher temperatures as the magnetic field increases, meaning that deconfinement and chiral symmetry restoration do not disentangle, at least in the explored range of external fields. The drop (rise) of the chiral condensate (Polyakov loop) at the transition seems sharper and sharper as the magnetic field increases, meaning that the (pseudo)transition is strengthening.

Such facts are confirmed by Figs. 4 and 5, where the susceptibilities of the chiral condensate and of the Polyakov loop are reported, for different values of the magnetic field: the peaks move to higher $\beta$ (hence to higher $T$ ) and become sharper and sharper as $B$ increases, i.e. their height increases and their width decreases. The increased strength of the transition is also appreciable from the plaquette (pure gauge action) distribution at the pseudocritical coupling, which is reported in Fig. 6 and which seems to evolve towards a double peak distribution, typical of a first order transition, as $B$ increases. Such hints for a change in the nature of the transition, however, have not yet been confirmed by simulations on larger lattices. Regarding the increase in pseudo-critical temperature, we notice that it is quite modest in magnitude and of the order of $2 \%$ at $|e| B \sim 1 \mathrm{GeV}^{2}$.

After the exploratory study of Ref. [57], new studies have appeared in the literature, which have added essential information and also opened new interesting questions. Let us first consider the investigation reported in [62]. There are three essential differences, with respect to [57], in the lattice discretization and approximation of QCD:

1) still within a rooted staggered fermion formulation, improved gauge (tree level Symanzik improved) and fermionic (stout link) discretizations have been implemented, and different lattice spacings have been explored, in order to get control over the continuum limit;

2) the authors have explored $N_{f}=2+1$ QCD, i.e. they have considered strange quark effects;

3) a physical quark mass spectrum has been adopted. 


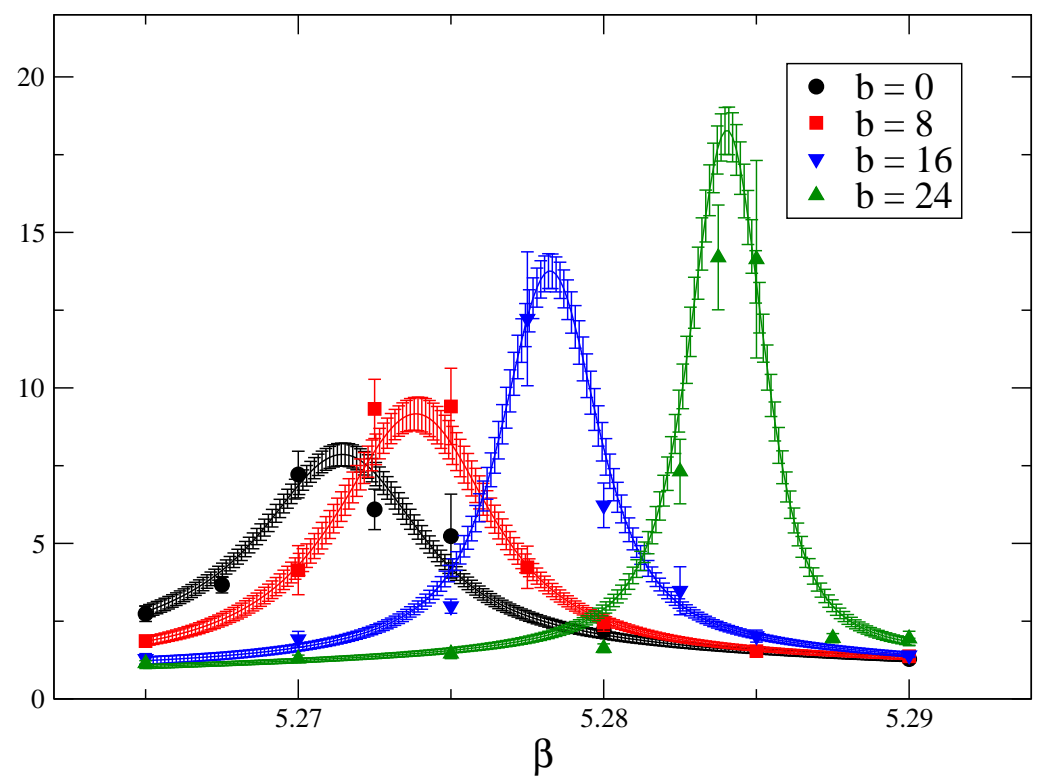

Fig. 4 Disconnected susceptibility of the chiral condensate as a function of the inverse gauge coupling $\beta$ and for different magnetic field quanta $b$, on a $16^{3} \times 4$ lattice and for a pion mass $m_{\pi} \sim 200 \mathrm{MeV}$. Figure taken from [57].

Concerning results, instead, the most striking difference with respect to [57] is that the pseudocritical temperature decreases, instead of increasing, in presence of the magnetic background, in particular it becomes 10-20\% lower for $|e| B \sim 1 \mathrm{GeV}^{2}$. The authors of [62] have put the decrease of the critical temperature in connection with another unexpected phenomenon that they observe, namely inverse magnetic catalysis, i.e. the fact that, at high enough temperatures, the chiral condensate starts decreasing, instead of increasing, as a function of $B$. A possibility pointed out in [65] is that inverse catalysis may originate from the gluonic sector, i.e. the distribution of gluon fields may change, as an indirect effect of the magnetic field mediated by quark loops, so as to destroy the chiral condensate. We point out that the study of [59] has shown instead that, at least at zero temperature, the modified distribution of gluon fields contributes to increase magnetic catalysis (dynamical contribution), however the situation may indeed be quite different around and above the deconfinement temperature.

Other aspects pointed out in [57] have instead been confirmed by [62]: in particular the absence of a clear splitting between deconfinement and chiral symmetry restoration, induced by the external field, and an increased strength of the transition 


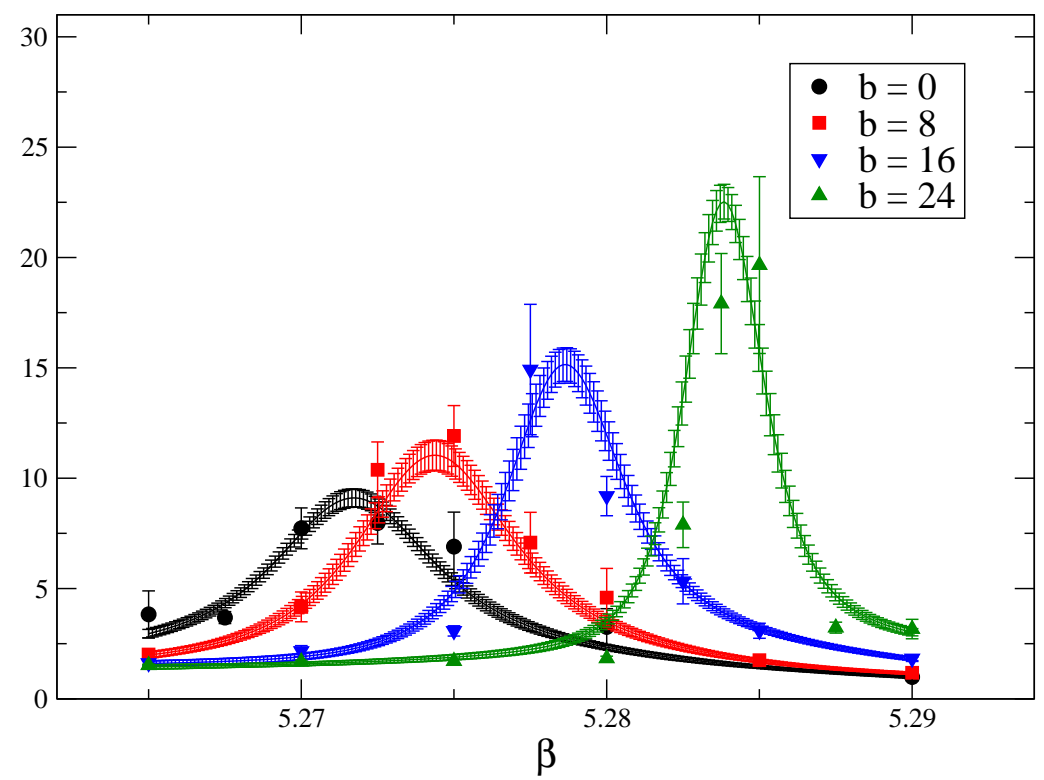

Fig. 5 Polyakov loop susceptibility as a function of the inverse gauge coupling $\beta$ and for different magnetic field quanta $b$, on a $16^{3} \times 4$ lattice and for a pion mass $m_{\pi} \sim 200 \mathrm{MeV}$. Figure taken from [57].

at $B \neq 0$, even without evidence for a change of its nature.

A different approach has been followed by the authors of Ref. [63]: they have explored 2 color QCD, making use of a discretization very close to that adopted in [57], i.e. with the same gauge and staggered action and a similar range of pion masses. However, to avoid the possible systematic effects connected with taking the square or fourth root of the fermionic determinant, they have considered a theory with four degenerate flavors, $N_{f}=4$, all carrying the same mass and electric charge. Their results are in quite good agreement with those of [57]: deconfinement and chiral symmetry restoration do not split; the transition gets sharper; the pseudocritical temperature increases as a function of $B$ and no inverse catalysis is observed, i.e. the chiral condensate is an increasing function of $B$ for all explored temperatures. The increase in $T_{C}$ is also larger than what observed in [57], being of the order of $10 \%$ at $|e| B \sim \mathrm{GeV}^{2}$ : that can possibly be explained by the fact that the authors of Ref. [63] make use of 4 flavors, instead of 2, moreover all carrying the charge of the $u$ quark, hence the influence of the magnetic field on the system can be larger.

Another interesting aspect, explored in [63], regards the actual fate of chiral symmetry above the transition and in presence of the background field. The authors have 


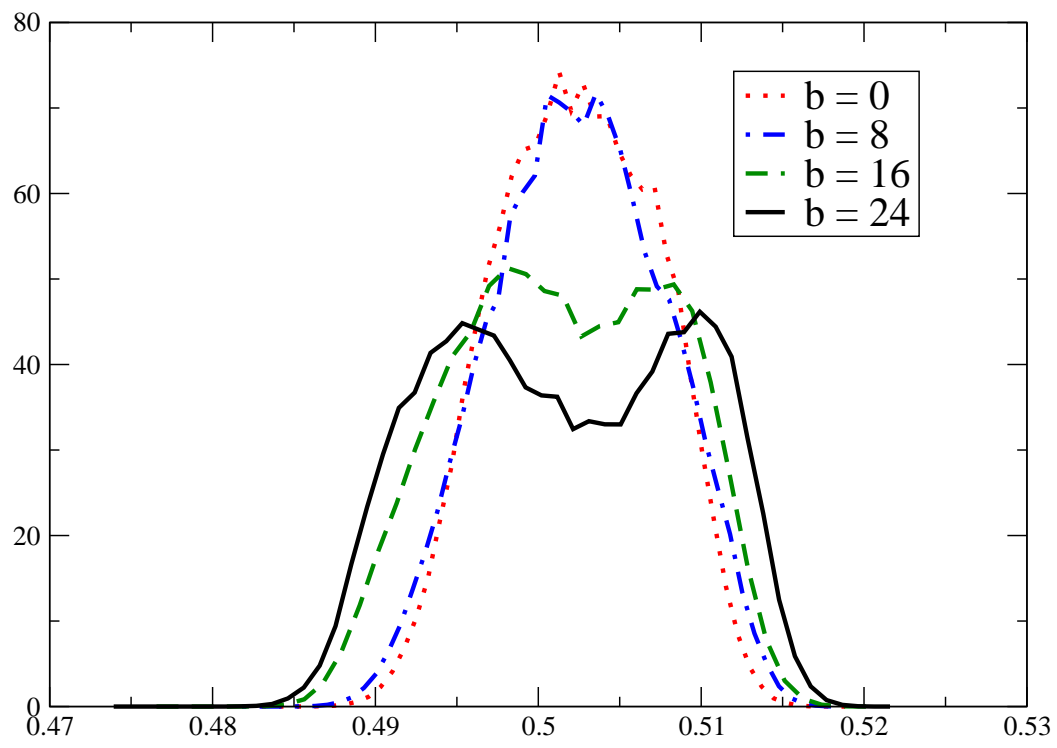

Fig. 6 Plaquette distribution at the pseudocritical temperature for $m_{\pi} \sim 200 \mathrm{MeV}$ and for various different values of the magnetic field. Figure taken from [57].

explicitly verified, by performing an extrapolation to the chiral limit, that the chiral condensate vanishes in the high temperature phase, i.e. that chiral symmetry is exact above $T_{c}$, also in presence of the magnetic backgroud. It is interesting to notice that, in the system explored in [63], the magnetic field does not break any of the flavor symmetries of the theory, since all quarks carry the same electric charge. However also in the standard case, in which quarks carry different charges, there are still diagonal chiral flavor generators which commute with the electric charge matrix and are not broken by the introduction of an external electromagnetic background field, so that an investigation similar to that performed in [63] should be done, to enquire about the actual realization of the unbroken symmetries above $T_{c}$.

To summarize, present lattice investigations about the influence of a strong magnetic field on the QCD phase diagram have given consistent indications about two facts: a magnetic field increases the strength of the QCD transition, however it does not change its nature, at least for $|e| B$ up to $1 \mathrm{GeV}^{2}$; in the same range of external fields, no significant splitting of deconfinement and chiral symmetry restoration has been detected.

There is a controversial issue, instead, regarding the location of the pseudocritical temperature, $T_{c}$, which increases as a function of $|e| B$ according to the results of [57, 63], while it decreases according to the results of [62]. It is perfectly possi- 


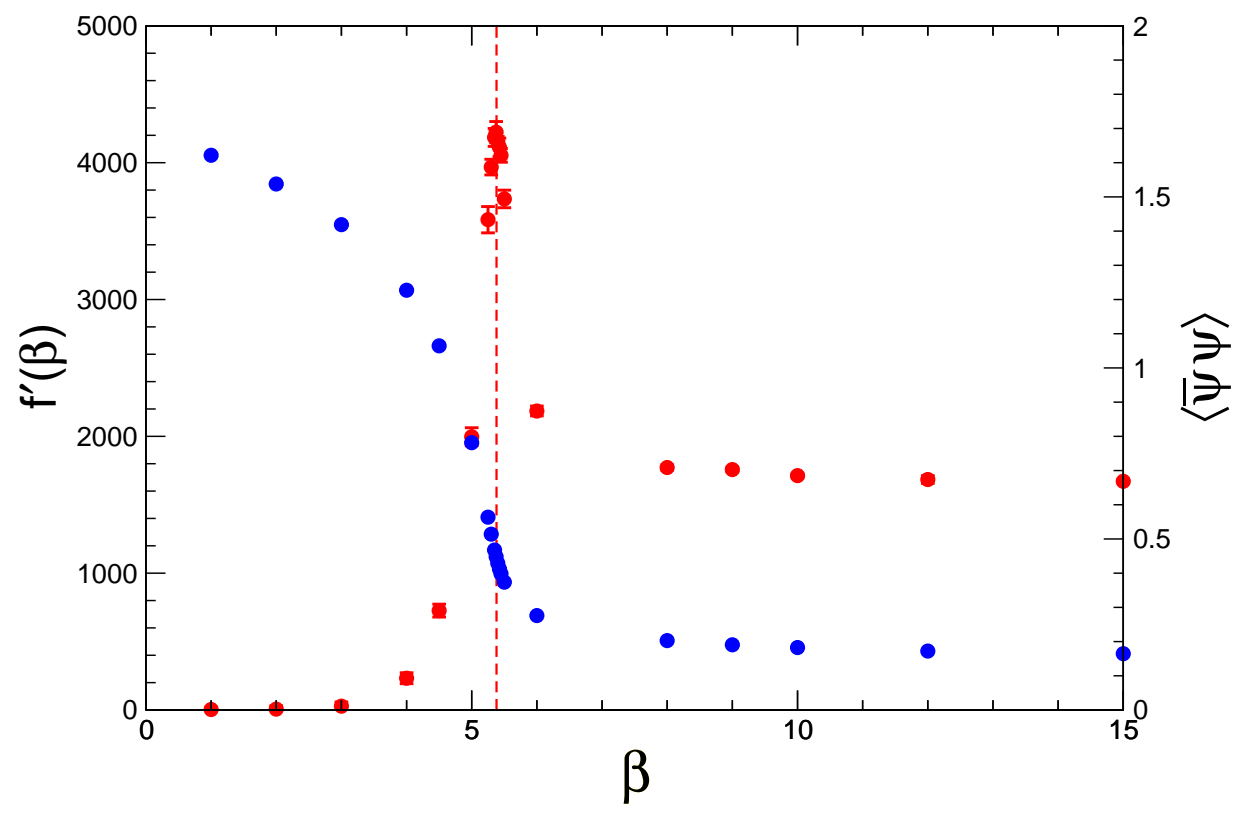

Fig. 7 Peak of the derivative of the free energy of the background field with respect to the inverse gauge coupling, together with the chiral condensate, for $N_{f}=2 \mathrm{QCD}$ and for a background chromomagnetic field $g B=\pi /\left(16 a^{2}\right) \sim 0.35 \mathrm{GeV}^{2}$. Figure taken from [49].

ble that various improvements regarding the lattice discretization may turn the very slowly increasing function $T_{c}(B)$ determined in [57] into a decreasing function. The main point is to understand which aspect is more directly related to the change of behavior and to the appearance of inverse catalysis. Is it just a problem of lattice discretization and approach to the continuum limit, or can the difference be traced back to the larger pion mass used in [57]? Could instead the introduction of the strange quark explain the differences? We believe that a clear answer to those questions can be given by considering each single aspect separately, and that the answer will be important by itself, since it will help clarifying the origin of unexpected behaviors such as inverse magnetic catalysis. 


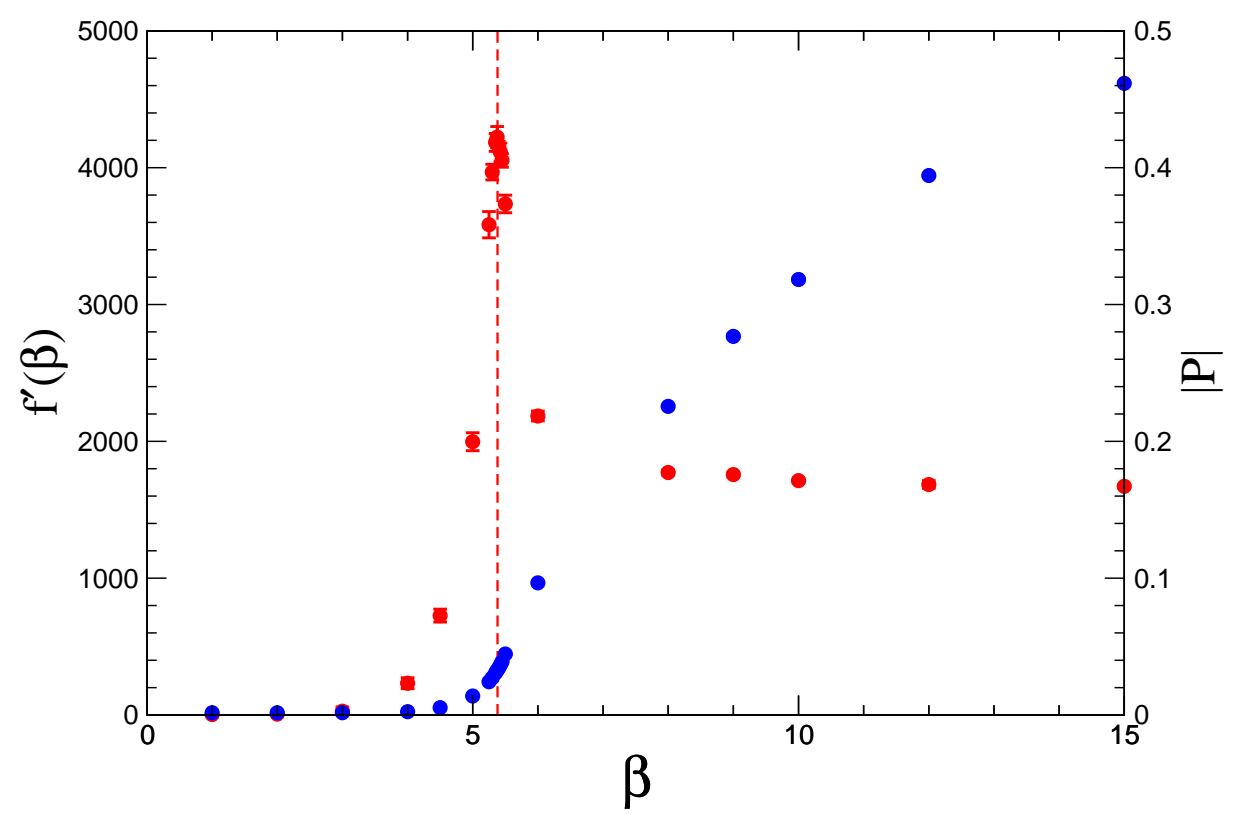

Fig. 8 Peak of the derivative of the free energy of the background field with respect to the inverse gauge coupling, the Polyakov loop, for $N_{f}=2 \mathrm{QCD}$ and for a background chromomagnetic field $g B=\pi /\left(16 a^{2}\right) \sim 0.35 \mathrm{GeV}^{2}$. Figure taken from [49].

\subsection{Deconfinement Transition in a Chromomagnetic Background}

A chromomagnetic background field influences directly gluodynamics, hence it makes sense to investigate its effects also in pure gauge simulations. A standard approach to introduce colored background fields has been described in Section 2.2 and is based on the formalism of the lattice Schrödinger functional [43, 44]]: such approach has been adopted in the literature to study the influence of background fields both in pure Yang-Mills theories and in full QCD, and for various kinds of background fields, going from those corresponding to a uniform magnetic field to those produced by magnetic monopoles [45, 46, 47, 48, 49, 50, 51]. In the following we shall consider the case of a constant background field [47, 48, 49].

Like a magnetic field, also a chromomagnetic background field leads to a shift of both chiral symmetry restoration, signalled by the drop of the chiral condensate, and of deconfinement, signalled by the rise of the Polyakov loop. The two transitions move together, moreover it is interesting to notice that the transition point coincides with the temperature at which the free energy (effective potential) of the background 


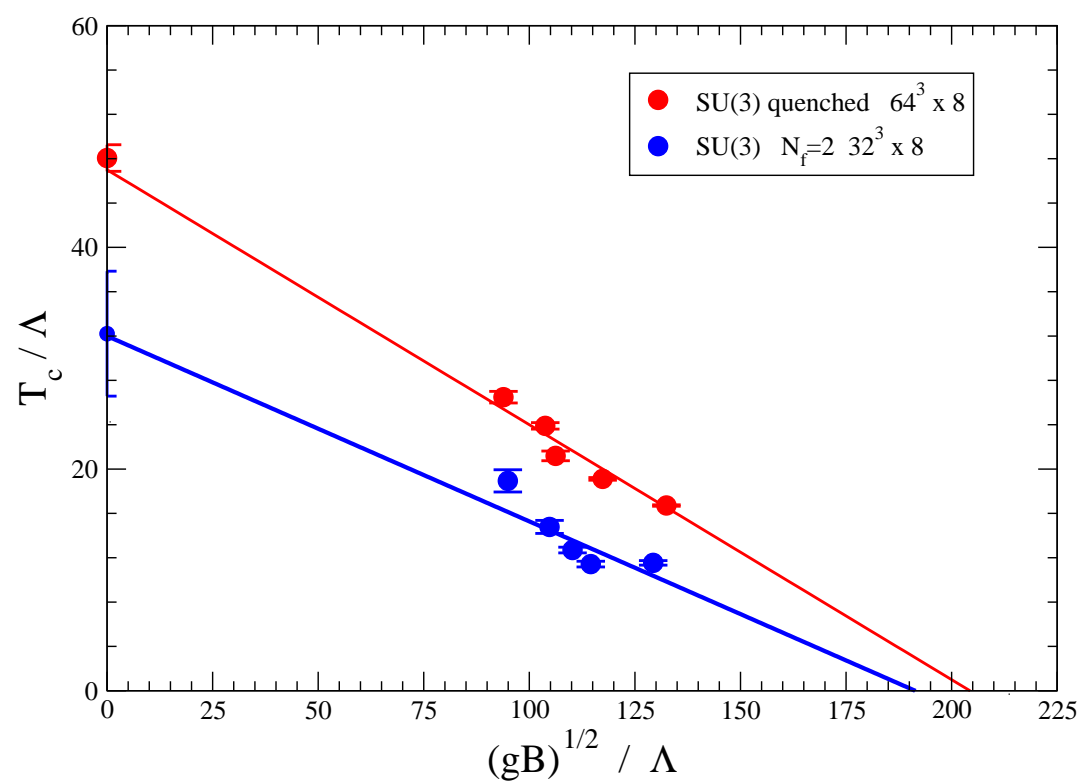

Fig. 9 Dependence of the critical temperature $T_{c}$ on the external chromomagnetic field for the pure gauge theory and for $N_{f}=2 \mathrm{QCD}$. Physical quantities are expressed in terms of the parameter $\Lambda \sim 6 \mathrm{MeV}$.

field shows a sudden change, the background field being screened (not screened) in the confined (deconfined) phase.

As an example, in Figs. 7 and 8 taken from [49], we show the behavior of the chiral condensate and of the Polyakov loop versus the inverse gauge coupling, $\beta$, together with the derivative of the free energy with respect to $\beta, f^{\prime}(\beta)$, which shows a sharp peak at the same point at which chiral symmetry restoration and deconfinement take place. Results make reference to simulations of $N_{f}=2 \mathrm{QCD}$ on a $32^{3} \times 8$ lattice, with a standard pure gauge and staggered fermion formulation, a lattice spacing $a \simeq 0.15 \mathrm{fm}$ and a bare quark mass $a m=0.075$; the value of the magnetic background field is $g B=\pi /\left(16 a^{2}\right) \sim 0.35 \mathrm{GeV}^{2}$.

Present lattice results indicate that, both for pure gauge and full QCD, the transition temperature decreases in presence of a constant background chromomagnetic field. This is shown in Fig. 9 [49], where the critical temperature $T_{c}$ (expressed in units of the parameter $\Lambda \sim 6 \mathrm{MeV}$ ), is shown as a function of $g B$, both for the pure gauge theory and $N_{f}=2 \mathrm{QCD}$. The change in the critical temperature is, in general, much larger than what happens in presence of an electromagnetic background: this can be interpreted in terms of the fact that a colored background field directly affects gluodynamics. An extrapolation of lattice results would even hint, as shown 


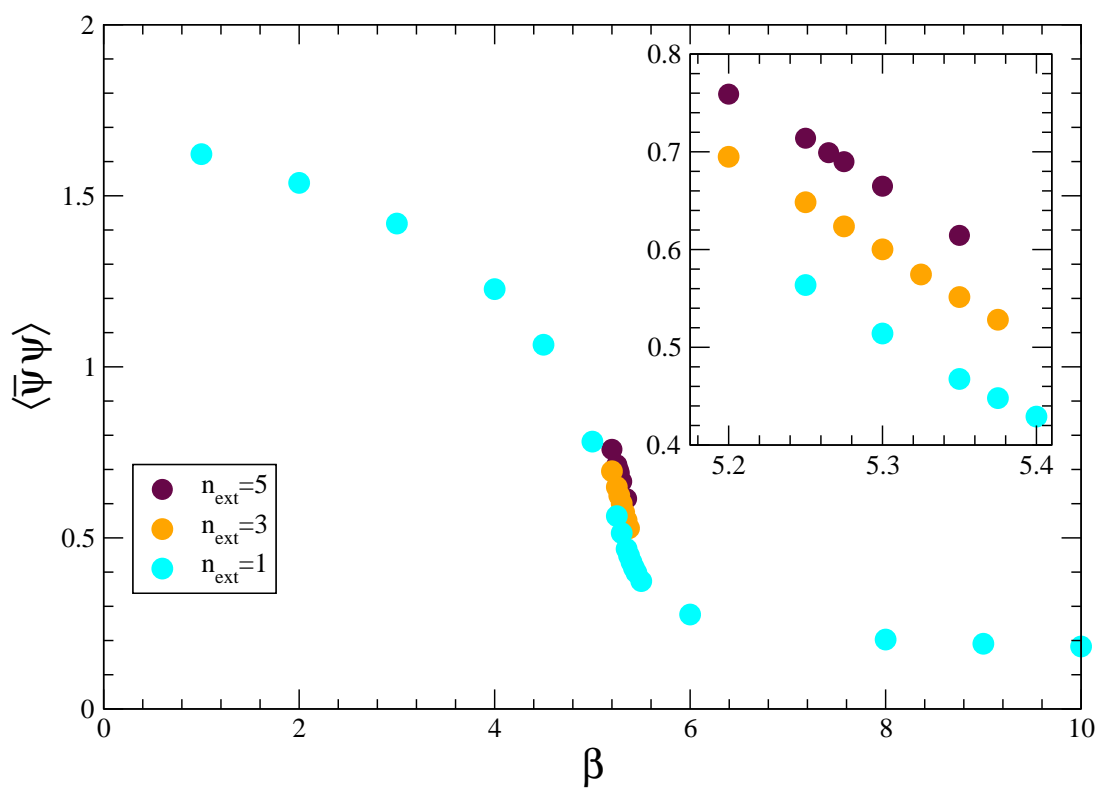

Fig. 10 Chiral condensate as a function of the inverse gauge coupling $\beta$ for different values of the external chromomagnetic field $g B=\pi n_{\text {ext }} /\left(16 a^{2}\right)$ and for $N_{f}=2$ QCD. Figure taken from [49].

in Fig. 9, at the presence of a zero temperature deconfining transition, for $\sqrt{g B}$ of the order of $1 \mathrm{GeV}$. It would be interesting to investigate this possibility further, in the future, for the possible cosmological and astrophysical implications it could have. One should repeat the study of [49] in presence of more physical quark masses (with the values adopted in [49], $m_{\pi}$ is of the order of $400-500 \mathrm{MeV}$ ) and closer to the continuum limit, also in order to reach higher values of the external field.

As a final comment, we notice that [49] also reports evidence about magnetic catalysis induced by the chromomagnetic field. Fig. 10, in particular, shows the behavior of the chiral condensate for a few values of $g B$ : it is clear that, at least around the transition, where data for all external fields are available, the chiral condensate grows as a function of $g B$. It is interesting to notice that in this case $T_{c}$ decreases anyway, even in presence of normal catalysis, i.e. inverse catalysis does not seem to be a necessary condition for a decreasing critical temperature, at least in presence of finite quark masses. 


\section{More on Gauge Field Modifications in External Electromagnetic Fields}

The lattice results that we have discussed in the previous Sections show that an electromagnetic background field can have a strong influence not only on quark dynamics, but also on gluodynamics, even if the interaction is not direct but mediated by quark loop effects. That does not come completely unexpected, at least in the strongly interacting, non-perturbative regime. Let us summarize a few facts:

1) we have shown that large part of magnetic catalysis is due to the modification of the gauge field distribution (dynamical contribution), i.e. that the increment of chiral symmetry breaking is lower by about $40 \%$ if gauge field configurations are sampled without taking into account the background field [59];

2) it is known, from model computations, that a strong electromagnetic background can modify gluon screening properties and influence confinement dynamics, reducing the confinement scale [36, 75, 35]: such effects on gluodynamics have been proposed in [65] as a possible explanation for inverse catalyis;

3) lattice computations have also explicitly shown that quantities related to quark confinement, like the Polyakov loop, are influenced by a background electromagnetic field, not only around the transition but also well inside the confined and the deconfined phases (see as an example the data reported in Fig. 2);

4) the results of [63] have shown that the magnetic field can also induce an asymmetry in the non-Abelian plaquette values, especially around and above the transition, with a possible significant effect on the equation of state of the Quark-Gluon Plasma.

It is surely of great importance to study and understand these effects in a deeper and more systematic way. Is it possible, for instance, that electromagnetic background fields may induce chromoelectric or chromomagnetic background fields? That has been discussed in some recent model studies (see e.g. [75]) and could have important phenomenological consequences, both for cosmology and astrophysics and for heavy ion collisions. In the following we would like to discuss how lattice simulations could further contribute to the issue, with an accent on symmetry aspects.

Let us consider a uniform and constant magnetic background field. In order to understand some of the gluonic field modifications induced by it, it is interesting to observe that it breaks explicitly charge conjugation symmetry, and to ask how such breaking propagates to the gluon sector.

Charge conjugation on gluon fields means $A_{\mu} \rightarrow-A_{\mu}$ or, at the level of lattice gauge link variables, $U_{\mu} \rightarrow U_{\mu}^{*}$. Symmetry under it means that a gauge configuration $U_{\mu}(x)$ has the same path integral probability of its complex conjugate $U_{\mu}^{*}(x)$ :

$$
\mathscr{D} U \mathscr{P}[U]=\mathscr{D} U^{*} \mathscr{P}\left[U^{*}\right] .
$$

As a consequence, all gluonic gauge inviarant quantities, i.e. traces over closed parallel transports $W_{C}[U]$, including plaquettes and Wilson loops, must be real: 


$$
\begin{aligned}
\left\langle W_{C}[U]\right\rangle^{*} & =\left\langle W_{C}^{*}[U]\right\rangle=\left\langle W_{C}\left[U^{*}\right]\right\rangle=\int \mathscr{D} U \mathscr{P}[U] W_{C}\left[U^{*}\right] \\
& =\int \mathscr{D} U^{*} \mathscr{P}\left[U^{*}\right] W_{C}[U]=\int \mathscr{D} U \mathscr{P}[U] W_{C}[U]=\left\langle W_{C}[U]\right\rangle
\end{aligned}
$$

where the first equality holds if the path integral measure is real, the second express the fact that $W_{C}$ is a trace over a product of links, while the fourth is simply a change of integration variables. Eq. (32) may be violated by possible spontaneous symmetry breaking effects, which may affect some particular loops, as it happens for the Polyakov loop in the deconfined phase.

In presence of an electromagnetic background field, such property must be lost, since the breaking of charge conjugation symmetry will propagate from the quark to the gluonic sector: $\mathscr{P}[U] \neq \mathscr{P}\left[U^{*}\right]$ because of the contribution from the fermion determinant. Let us see that more explicitly, considering the particular case of the trace over a plaquette.

The most direct way to look for effective quark contributions to the gauge field distribution is to consider the loop expansion of the fermionic determinant. If we write the fermion matrix as $M=m_{f} \mathrm{Id}+D$, where $D$ is the discretization of the Dirac operator and $m_{f}$ is the bare quark mass, then the following formal expansion holds:

$$
\operatorname{det} M=\exp \left(\operatorname{Tr} \log \left(m_{f} \operatorname{Id}+D\right)\right) \propto \exp \left(-\sum_{k} \frac{(-1)^{k}}{k m_{f}^{k}} \operatorname{Tr} D^{k}\right)
$$

$D^{k}$ is made up of parallel transports connecting lattice sites; the trace operator implies that only closed parallel transports will contribute. Moreover, for each contributing loop, there is an equal contribution from its hermitian conjugate. If $D$ is a standard, nearest neighbour discretization, the first non-trivial term comes for $k=4$ and contains a coupling to the plaquette operator, which can be expressed as:

$$
\frac{\Delta \beta\left(m_{f}\right)}{N_{c}} \frac{1}{2}\left(\operatorname{Tr} \Pi_{\mu v}(x)+\operatorname{Tr} \Pi_{\mu v}^{\dagger}(x)\right)=\frac{\Delta \beta\left(m_{f}\right)}{N_{c}} \operatorname{Re} \operatorname{Tr} \Pi_{\mu v}(x)
$$

where $\Delta \beta\left(m_{f}\right)$ is a coefficient which depends on $m_{f}$ and on the particular fermion discretization adopted. The total effect can be viewed as a simple renormalization of the inverse bare gauge coupling, $\beta \rightarrow \beta+\sum_{f} \Delta \beta\left(m_{f}\right)$, where the sum runs over flavors.

Let us now consider the effect of an external electromagnetic field. That will change the definition of $D$, see e.g. Eq. (2), so that each loop contribution to the determinant will get a $U(1)$ phase from the external field. In particular, the expression in Eq. 34 for plaquettes will be modified into

$$
\begin{aligned}
& \frac{\Delta \beta\left(m_{f}\right)}{N_{c}} \frac{1}{2}\left(e^{\mathrm{i} \phi_{\mu v}(x)} \operatorname{Tr} \Pi_{\mu v}(x)+e^{-\mathrm{i} \phi_{\mu v}(x)} \operatorname{Tr} \Pi_{\mu v}^{\dagger}(x)\right)= \\
= & \frac{\Delta \beta\left(m_{f}\right)}{N_{c}}\left(\cos \left(\phi_{\mu v}(x)\right) \operatorname{Re} \operatorname{Tr} \Pi_{\mu v}(x)-\sin \left(\phi_{\mu v}(x)\right) \operatorname{Im} \operatorname{Tr} \Pi_{\mu v}(x)\right) .
\end{aligned}
$$


The phases $\phi_{\mu v}$ are in general non-trivial. For the particular case of a uniform magnetic field $B$ in the $\hat{z}$ direction we have $\phi_{x y}=q_{f} B a^{2}$, where $q_{f}$ is the quark charge. This implies that, apart from the case in which there is exact cancellation among quark flavors carrying equal masses and opposite electric charges (but this does not happen in real QCD), there will be a non-trivial coupling to the imaginary part of some plaquette traces, which will then develop a non-zero expectation value. Notice that this is exactly what happens also for the Polyakov loop in presence of an imaginary baryon chemical potential.

It is interesting to understand the meaning of a non-zero expectation value for the imaginary part of the trace of the plaquette, in terms of continuum quantities. Remember that, in the formal continuum limit, the plaquette operator is linked to the gauge field strenght $G_{\mu \nu}=G_{\mu \nu}^{a} T^{a}$ as follows:

$$
\Pi_{\mu \nu} \simeq \exp \left(\mathrm{i} G_{\mu v} a^{2}\right)
$$

Considering the expansion of the exponential on the right hand side, the first nontrivial contribution to the imaginary part of its trace comes from the third order, which indeed is proportional to

$$
-\mathrm{i} \operatorname{Tr} G_{\mu \nu}^{3}=-i G_{\mu \nu}^{a} G_{\mu \nu}^{b} G_{\mu \nu}^{c} \operatorname{Tr}\left(T^{a} T^{b} T^{c}\right)=\frac{1}{4}\left(f^{a b c}-\mathrm{i} d^{a b c}\right) G_{\mu \nu}^{a} G_{\mu \nu}^{b} G_{\mu \nu}^{c}
$$

where no sum over $\mu, v$ is understood. A non-zero expectation value for the imaginary part of the plaquette, therefore, corresponds to a non-zero charge odd, three gluon condensate, constructed in terms of the symmetric tensor $d^{a b c}$ :

$$
\left\langle d^{a b c} G_{\mu \nu}^{a} G_{\mu \nu}^{b} G_{\mu \nu}^{c}\right\rangle \neq 0 .
$$

Such condensate, which is zero by charge conjugation symmetry in the normal QCD vacuum, will develop a non-zero value along some directions, e.g. for $(\mu, v)=$ $(x, y)$ in case of a magnetic field in the $\hat{z}$ direction, and can be considered as a nontrivial, charge-odd gluon background induced by the external field. Of course the effect will be present only for $N_{c} \geq 3$ colors, since for $S U(2)$ all traces are real and indeed $d^{a b c}=0$.

The loop expansion of the determinant, examined above, is just a way to show explicitly that such effects can be present, but cannot be quantitative, since the expansion is purely formal (apart from the limit of large quark masses). A systematic investigation can and must be performed in the future by means of lattice simulations.

Similarly to charge conjugation symmetry, one can investigate electromagnetic backgrounds which break explicitly the symmetry under $\mathrm{CP}$, i.e. such that $\mathbf{E} \cdot \mathbf{B} \neq 0$, and ask how $\mathrm{CP}$ violation propagates to the gluon sector, giving rise to an effective $\theta$ parameter. Such phenomenon is in some sense complementary to the chiral magnetic effect and is related in general to the effective QED-QCD interactions in the pseudoscalar channel [100, 101, 102, 103]. It has been studied recently by a first exploratory lattice investigation [104], where numerical simulations at imaginary 
electric fields plus analytic continuation have been exploited to determine the susceptibility $\chi_{C P}$ of the QCD vacuum to CP-odd electromagnetic fields, defined by $\theta_{\text {eff }} \simeq \chi_{C P} e^{2} \mathbf{E} \cdot \mathbf{B}$, obtaining $\chi_{C P} \sim 7 \mathrm{GeV}^{-4}$ for QCD with two staggered flavors and $m_{\pi} \sim 480 \mathrm{MeV}$.

Finally, let us discuss a few other lines along which the effects of electromagnetic background fields on the gluon sector could be further investigated. The first consists in the determination of the effective action of given gluonic backgrounds, using the techniques described in Section 2.2 but in combination with non-trivial electromagnetic external fields: that would give the possibility of studying if the latter can give rise to instabilities in the gluon sector, leading to the generation of non-trivial gluonic backgrounds (as proposed e.g. in [75]). The second regards the measurement of glueball masses and screening masses in presence of magnetic backgrounds, which could give the possibility, for instance, to test the proposal given in [36] about the lowering of the confinement scale in presence of a strong magnetic field. Finally, it would be important to perform a careful investigation about the influence of magnetic backgrounds on the QCD equation of state, as proposed in [63].

\section{Conclusions}

Numerical simulations in presence of background external fields have been considered since the early stages of Lattice QCD. The last few years, however, have seen the development of considerable activity on the subject, which has been driven by the recent interest in theoretical and phenomenological issues regarding the behavior of strongly interacting matter in magnetic fields.

In this review, after a general overview about the formulation of lattice QCD in presence of magnetic or chromomagnetic background fields, we have discussed the present status of lattice studies regarding magnetic catalysis and the QCD phase diagram. In the low temperature phase, consistent results have been obtained, by different groups, which confirm magnetic catalysis and the predictions coming from chiral perturbation theory and some effective models. Good part of the enhancement of chiral symmetry breaking seems to be associated with modifications of gluon fields induced by the magnetic field via quark loops. A chromagnetic background induces magnetic catalysis as well.

In the high temperature phase, consistent results have been obtained regarding the fact that a magnetic or chromomagnetic field shifts both chiral symmetry restoration and deconfinement and that the two transitions do not split, at least for fields up to $O(1) \mathrm{GeV}^{2}$. A magnetic field also leads to a strengthening of the transition, which however does not seem to turn into a strong first order, at least for fields up to $O(1) \mathrm{GeV}^{2}$; it would be interesting however, to further investigate the issue also in combination with other external parameters, like a baryon chemical potential, since that could be relevant to the search for a critical endpoint in the QCD phase diagram.

Regarding the shift in the pseudocritical temperature induced by a background magnetic field, existing studies have shown that a modification of the lattice imple- 
mentation of QCD can change the outcome, going from a slight increase (of the order a few $\%$ for $|e| B$ of $O(1) \mathrm{GeV}^{2}$ ) to a decrease (of the order of $10 \%$ for $|e| B$ of $O(1) \mathrm{GeV}^{2}$ ) when an improved discretization and a physical quark mass spectrum are used; in the latter case one also observes inverse magnetic catalysis, i.e. a decrease of the chiral condensate as a function of $B$, in the high temperature, deconfined phase. A decrease of the pseudocritical temperature is also observed in presence of a chromomagnetic background field. A remaining question is which effect is more relevant to explain the discrepancy among different lattice studies, i.e. whether lattice artifacts, or the unphysical quark spectrum, or both are at its origin.

One of the aspects that emerges more clearly from present studies is the fact that gluon fields are strongly modified by external electromagnetic fields, even though indirectly, by means of dynamical quark loops. We believe that this aspect can and should be investigated more sistematically by lattice simulations: in Sec. 5]we have discussed and suggested a few possible ways to do that by future studies.

Acknowledgements The author is grateful to P. Cea, L. Cosmai, M. Mariti, S. Mukherjee, F. Negro and F. Sanfilippo for collaboration on some of topics discussed in this review. He also acknowledges M. Chernodub, G. Endrodi, E. Fraga, K. Fukushima, V. Miransky and M. Ruggieri for many useful discussions.

\section{References}

1. T. Vachaspati, Phys. Lett. B 265, 258 (1991).

2. D. E. Kharzeev, L. D. McLerran and H. J. Warringa, Nucl. Phys. A 803, 227 (2008).

3. V. Skokov, A. Y. Illarionov and V. Toneev, Int. J. Mod. Phys. A 24, 5925 (2009).

4. A. Vilenkin, Phys. Rev. D 22, 3080 (1980).

5. K. Fukushima, D. E. Kharzeev and H. J. Warringa, Phys. Rev. D 78, 074033 (2008).

6. P. Cea, Int. J. Mod. Phys. D 13, 1917 (2004).

7. R. C. Duncan and C. Thompson, Astrophys. J. 392, L9 (1992).

8. S. Mereghetti, Astron. Astrophys. Rev. 15, 225-287 (2008).

9. A. Salam and J. A. Strathdee, Nucl. Phys. B 90, 203 (1975).

10. A. D. Linde, Phys. Lett. B 62, 435 (1976).

11. S. Kawati, G. Konisi, H. Miyata, Phys. Rev. D28, 1537-1541 (1983).

12. S. P. Klevansky and R. H. Lemmer, Phys. Rev. D 39, 3478 (1989).

13. H. Suganuma and T. Tatsumi, Annals Phys. 208, 470 (1991).

14. K. G. Klimenko, Z. Phys. C 54, 323 (1992); Theor. Math. Phys. 94, 393 (1993).

15. S. Schramm, B. Muller, A. J. Schramm, Mod. Phys. Lett. A7, 973-982 (1992).

16. K. G. Klimenko, B. V. Magnitsky, A. S. Vshivtsev, Nuovo Cim. A107, 439-452 (1994).

17. V. P. Gusynin, V. A. Miransky and I. A. Shovkovy, Phys. Rev. Lett. 73, 3499 (1994) [Erratumibid. 76, 1005 (1996)].

18. V. P. Gusynin, V. A. Miransky and I. A. Shovkovy, Phys. Lett. B 349, 477 (1995).

19. I. A. Shushpanov and A. V. Smilga, Phys. Lett. B 402, 351 (1997).

20. T. D. Cohen, D. A. McGady and E. S. Werbos, Phys. Rev. C 76, 055201 (2007).

21. A. Y. .Babansky, E. V. Gorbar, G. V. Shchepanyuk, Phys. Lett. B419, 272-278 (1998).

22. D. Ebert, K. G. Klimenko, M. A. Vdovichenko and A. S. Vshivtsev, Phys. Rev. D 61, 025005 (2000).

23. A. Goyal, M. Dahiya, Phys. Rev. D D62, 025022 (2000).

24. N. O. Agasian and I. A. Shushpanov, Phys. Lett. B 472, 143 (2000). 
25. D. Ebert, V. V. Khudyakov, V. C. Zhukovsky, K. G. Klimenko, Phys. Rev. D65, 054024 (2002).

26. D. N. Kabat, K. -M. Lee, E. J. Weinberg, Phys. Rev. D66, 014004 (2002).

27. V. G. Filev, C. V. Johnson, R. C. Rashkov and K. S. Viswanathan, JHEP 0710, 019 (2007) [hep-th/0701001].

28. T. Albash, V. G. Filev, C. V. Johnson and A. Kundu, JHEP 0807, 080 (2008) [arXiv:0709.1547 [hep-th]].

29. C. V. Johnson and A. Kundu, JHEP 0812, 053 (2008).

30. E. Rojas, A. Ayala, A. Bashir, A. Raya, Phys. Rev. D77, 093004 (2008).

31. O. Bergman, G. Lifschytz and M. Lippert, Phys. Rev. D 79, 105024 (2009).

32. A. V. Zayakin, JHEP 0807, 116 (2008).

33. K. Fukushima and J. M. Pawlowski, arXiv:1203.4330 [hep-ph].

34. M. S. Alam, V. S. Kaplunovsky and A. Kundu, JHEP 1204, 111 (2012) [arXiv:1202.3488 [hep-th]].

35. I. A. Shovkovy, arXiv:1207.5081 [hep-ph].

36. V. A. Miransky and I. A. Shovkovy, Phys. Rev. D 66, 045006 (2002).

37. F. Preis, A. Rebhan and A. Schmitt JHEP 1103, 033 (2011); J. Phys. G G 39, 054006 (2012); arXiv: 1209.4468 [hep-ph].

38. G. Martinelli, G. Parisi, R. Petronzio and F. Rapuano, Phys. Lett. B 116, 434 (1982).

39. C. W. Bernard, T. Draper, K. Olynyk and M. Rushton, Phys. Rev. Lett. 49, 1076 (1982).

40. W. Detmold, B. C. Tiburzi and A. Walker-Loud, Phys. Rev. D 73, 114505 (2006) [heplat/0603026]; Phys. Rev. D 79, 094505 (2009) [arXiv:0904.1586 [hep-lat]]; Phys. Rev. D 81, 054502 (2010) [arXiv:1001.1131 [hep-lat]].

41. B. C. Tiburzi, PoS LATTICE 2011, 020 (2011) [arXiv:1110.6842 [hep-lat]]; Nucl. Phys. A 814, 74 (2008) [arXiv:0808.3965 [hep-ph]]; Phys. Lett. B 674, 336 (2009) [arXiv:0809.1886 [hep-lat]].

42. A. Alexandru and F. X. Lee, PoS LATTICE 2008, 145 (2008) [arXiv:0810.2833 [hep-lat]]; PoS LAT 2009, 144 (2009) [arXiv:0911.2520 [hep-lat]].

43. M. Luscher, R. Narayanan, P. Weisz and U. Wolff, Nucl. Phys. B 384, 168 (1992).

44. M. Luscher and P. Weisz, Nucl. Phys. B 452, 213 (1995).

45. P. Cea and L. Cosmai, Phys. Lett. B 264, 415 (1991).

46. P. Cea, L. Cosmai and A. D. Polosa, Phys. Lett. B 392, 177 (1997).

47. P. Cea and L. Cosmai, JHEP 0302, 031 (2003).

48. P. Cea and L. Cosmai, JHEP 0508, 079 (2005).

49. P. Cea, L. Cosmai and M. D'Elia, JHEP 0712, 097 (2007); PoS LAT 2006, 062 (2006) [heplat/0610014]; PoS LAT 2007, 295 (2007) [arXiv:0710.1449 [hep-lat]].

50. S. Antropov, M. Bordag, V. Demchik and V. Skalozub, Int. J. Mod. Phys. A 26, 4831 (2011).

51. P. Cea, L. Cosmai and M. D'Elia, JHEP 0402, 018 (2004).

52. M. Abramczyk, T. Blum, G. Petropoulos and R. Zhou, PoS LAT 2009, 181 (2009).

53. P. V. Buividovich, M. N. Chernodub, E. V. Luschevskaya and M. I. Polikarpov, Phys. Rev. D 80, 054503 (2009), Phys. Lett. B 682, 484 (2010).

54. P. V. Buividovich, M. N. Chernodub, E. V. Luschevskaya and M. I. Polikarpov, Nucl. Phys. B 826, 313 (2010) [arXiv:0906.0488 [hep-lat]].

55. P. V. Buividovich, M. N. Chernodub, E. V. Luschevskaya and M. I. Polikarpov, Phys. Rev. D 81, 036007 (2010).

56. P. V. Buividovich, M. N. Chernodub, D. E. Kharzeev, T. Kalaydzhyan, E. V. Luschevskaya and M. I. Polikarpov, Phys. Rev. Lett. 105, 132001 (2010).

57. M. D'Elia, S. Mukherjee, F. Sanfilippo, Phys. Rev. D 82, 051501 (2010).

58. V. V. Braguta, P. V. Buividovich, T. Kalaydzhyan, S. V. Kuznetsov, M. I. Polikarpov, PoS LATTICE2010, 190 (2010). [arXiv:1011.3795 [hep-lat]].

59. M. D'Elia and F. Negro, Phys. Rev. D 83, 114028 (2011).

60. V. V. Braguta, P. V. Buividovich, M. N. Chernodub and M. I. Polikarpov, arXiv:1104.3767 [hep-lat].

61. A. Yamamoto, Phys. Rev. Lett. 107, 031601 (2011). 
62. G. S. Bali, F. Bruckmann, G. Endrodi, Z. Fodor, S. D. Katz, S. Krieg, A. Schafer and K. K. Szabo, JHEP 1202, 044 (2012).

63. E. -M. Ilgenfritz, M. Kalinowski, M. Muller-Preussker, B. Petersson and A. Schreiber, Phys. Rev. D 85, 114504 (2012).

64. E. V. Luschevskaya and O. V. Larina, arXiv:1203.5699 [hep-lat].

65. G. S. Bali, F. Bruckmann, G. Endrodi, Z. Fodor, S. D. Katz and A. Schafer, arXiv:1206.4205 [hep-lat].

66. G. S. Bali, F. Bruckmann, M. Constantinou, M. Costa, G. Endrodi, S. D. Katz, H. Panagopoulos and A. Schaefer, arXiv:1209.6015 [hep-lat].

67. K. G. Wilson, Phys. Rev. D 10, 2445 (1974).

68. G. 't Hooft, Nucl. Phys. B 153, 141 (1979).

69. J. Smit and J. C. Vink, Nucl. Phys. B 286, 485 (1987).

70. P. H. Damgaard and U. M. Heller, Nucl. Phys. B 309, 625 (1988).

71. M. H. Al-Hashimi and U. J. Wiese, Annals Phys. 324, 343 (2009).

72. E. Shintani, S. Aoki, N. Ishizuka, K. Kanaya, Y. Kikukawa, Y. Kuramashi, M. Okawa and A. Ukawa et al., Phys. Rev. D 75, 034507 (2007).

73. T. Banks, A. Casher, Nucl. Phys. B169, 103 (1980).

74. R. Gatto and M. Ruggieri, arXiv:1207.3190 [hep-ph].

75. B. V. Galilo and S. N. Nedelko, Phys. Rev. D 84, 094017 (2011).

76. K. Fukushima and Y. Hidaka, arXiv:1209.1319 [hep-ph].

77. N. O. Agasian and S. M. Fedorov, Phys. Lett. B 663, 445 (2008).

78. E. S. Fraga and A. J. Mizher, Phys. Rev. D 78, 025016 (2008).

79. J. K. Boomsma and D. Boer, Phys. Rev. D 81, 074005 (2010).

80. K. Fukushima, M. Ruggieri and R. Gatto, Phys. Rev. D 81, 114031 (2010).

81. A. J. Mizher, M. N. Chernodub and E. S. Fraga, Phys. Rev. D 82, 105016 (2010).

82. R. Gatto and M. Ruggieri, Phys. Rev. D 82, 054027 (2010).

83. S. -i. Nam, C. -W. Kao, Phys. Rev. D 83, 096009 (2011).

84. J. O. Andersen and R. Khan, Phys. Rev. D 85, 065026 (2012).

85. S. .Fayazbakhsh and N. Sadooghi, Phys. Rev. D 83, 025026 (2011).

86. N. Evans, T. Kalaydzhyan, K. -y. Kim and I. Kirsch, JHEP 1101, 050 (2011).

87. R. Gatto and M. Ruggieri, Phys. Rev. D 83, 034016 (2011).

88. A. Rabhi and C. Providencia, Phys. Rev. C 83, 055801 (2011).

89. K. Kashiwa, Phys. Rev. D 83, 117901 (2011).

90. B. Chatterjee, H. Mishra and A. Mishra, Phys. Rev. D 84, 014016 (2011).

91. V. Skokov, Phys. Rev. D 85, 034026 (2012).

92. E. S. Fraga and L. F. Palhares, arXiv:1201.5881 [hep-ph].

93. S. S. Avancini, D. P. Menezes, M. B. Pinto and C. Providencia, Phys. Rev. D 85, 091901 (2012).

94. J. O. Andersen and A. Tranberg, arXiv:1204.3360 [hep-ph].

95. G. N. Ferrari, A. F. Garcia and M. B. Pinto, arXiv:1207.3714 [hep-ph].

96. E. S. Fraga, J. Noronha and L. F. Palhares, arXiv:1207.7094 [hep-ph].

97. M. N. Chernodub, Phys. Rev. D 82, 085011 (2010); Phys. Rev. Lett. 106, 142003 (2011).

98. Y. Hidaka and A. Yamamoto, arXiv:1209.0007 [hep-ph].

99. M. N. Chernodub, arXiv:1209.3587 [hep-ph].

100. M. M. Musakhanov and F. C. Khanna, hep-ph/9605232.

101. H. T. .Elze and J. Rafelski, In *Sandansky 1998, Frontier tests of QED and physics of the vacuum* 425-439 [hep-ph/9806389].

102. H. T. .Elze, B. Muller and J. Rafelski, hep-ph/9811372.

103. M. Asakawa, A. Majumder and B. Muller, Phys. Rev. C 81, 064912 (2010).

104. M. D'Elia, M. Mariti and F. Negro, arXiv:1209.0722 [hep-lat]. 